\title{
Spatial and identity negative priming in audition: Evidence of feature binding in auditory spatial memory
}

\author{
Susanne Mayr • Axel Buchner • Malte Möller • \\ Robert Hauke
}

Published online: 18 May 2011

(C) Psychonomic Society, Inc. 2011

\begin{abstract}
Two experiments are reported with identical auditory stimulation in three-dimensional space but with different instructions. Participants localized a cued sound (Experiment 1) or identified a sound at a cued location (Experiment 2). A distractor sound at another location had to be ignored. The prime distractor and the probe target sound were manipulated with respect to sound identity (repeated vs. changed) and location (repeated vs. changed). The localization task revealed a symmetric pattern of partial repetition costs: Participants were impaired on trials with identity-location mismatches between the prime distractor and probe target - that is, when either the sound was repeated but not the location or vice versa. The identification task revealed an asymmetric pattern of partial repetition costs: Responding was slowed down when the prime distractor sound was repeated as the probe target, but at another location; identity changes at the same location were not impaired. Additionally, there was evidence of retrieval of incompatible prime responses in the identification task. It is concluded that feature binding of auditory prime distractor information takes place regardless of whether the task is to identify or locate a sound. Instructions determine the kind of identity-location mismatch that is detected. Identity information predominates over location information in auditory memory.
\end{abstract}

S. Mayr $\cdot$ A. Buchner $\cdot$ M. Möller $\cdot$ R. Hauke

Heinrich-Heine-Universität,

Düsseldorf, Germany

\section{S. Mayr $(\bowtie)$}

Institut für Experimentelle Psychologie,

Heinrich-Heine-Universität,

40225 Düsseldorf, Germany

e-mail: susanne.mayr@uni-duesseldorf.de
Keywords Audition - Attention: space-based - Attention: object-based

Negative priming - the slowed-down responding to previously ignored information-was originally discovered and studied in the visual modality (for reviews, see Fox, 1995; Tipper, 2001) in order to understand how human information processing deals with distracting information in the course of goal-directed behavior. However, by now, the negative priming effect has also become a well-established phenomenon in the auditory modality (Banks, Roberts, \& Ciranni, 1995; Buchner \& Mayr, 2004; Buchner, Zabal, \& Mayr, 2003; Leboe, Mondor, \& Leboe, 2006; Mayr \& Buchner, 2006, 2010a; Mayr, Buchner, \& Dentale, 2009a; Mondor, Leboe, \& Leboe, 2005; Zabal \& Buchner, 2006). By and large, auditory negative priming follows the same principles as its visual counterpart (Mayr \& Buchner, 2007), which is by no means a trivial finding. In vision, attentional selection is supported by peripheral mechanisms such as head movements and shifting fixations. In contrast, auditory central selection (as measured by negative priming) has to be accomplished without peripheral support (Banks et al., 1995), which might result in either a stronger or a differently organized selection mechanism in the auditory, as compared with the visual, modality. All of the cited auditory studies focused on identity negative priming and showed that participants were slowed down in responding to a probe sound's identity when this sound identity had to be ignored in the prime. Comparably, identity negative priming in the visual modality is found when participants are slowed down in responding to the identity of a visual object (such as a line drawing) that had to be ignored previously (e.g., Tipper, 1985). Identity negative priming, irrespective of stimulus modality, is usually explained either by inhibition of the prime 
distractor, which has to be overcome when the previously ignored stimulus becomes the probe target (Tipper, 1985, 2001; Tipper \& Cranston, 1985), or by retrieval of prime episode information that is incompatible with probe responding (Mayr \& Buchner, 2006; Neill \& Valdes, 1992; Neill, Valdes, Terry, \& Gorfein, 1992; Rothermund, Wentura, \& De Houwer, 2005; for evidence of a very similar process of episodic retrieval in the visual search paradigm, see Huang, Holcombe, \& Pashler, 2004).

The counterpart to identity negative priming is the spatial negative priming effect. In a spatial priming task, participants have to select an object on the basis of its identity or an aspect thereof (e.g., object color or shape) and have to report the location at which the selected object has been presented, while ignoring objects (and their locations) that do not conform to the selection criterion (e.g., Tipper, Brehaut, \& Driver, 1990). On so-called ignored repetition trials in a standard visuospatial priming task, the attended probe target object is presented at the same position as the previously ignored prime distractor. A spatial negative priming effect is found when responses to ignored repetition trials are slowed down, in comparison with control trials in which there is no location repetition between any of the two prime and probe stimuli. In other words, spatial negative priming is the aftereffect of ignoring a task-irrelevant spatial position in favor of attending and responding to another, task-relevant position. The majority of studies concerned with spatial negative priming in the visual modality have revealed that responding to previously irrelevant locations is indeed impaired (see, e.g., Buckolz, Goldfarb, \& Khan, 2004; Chao, 2009; Milliken, Tipper, Houghton, \& Lupiáñez, 2000; Milliken, Tipper, \& Weaver, 1994; Neill, Terry, \& Valdes, 1994; Neill et al., 1992; Tipper et al., 1990; Tipper, Weaver, \& Milliken, 1995).

The spatial negative priming task can be regarded as a useful tool for investigating how human information processing deals with objects appearing at to-be-ignored locations while task-relevant objects are presented at other locations. Originally, and in analogy to identity negative priming, spatial negative priming has been attributed to inhibition of ignored locations during prime processing (Tipper et al., 1990) or to retrieval of probe-incompatible prime episode information (i.e., do-not-respond tags) associated with the ignored location (Neill \& Valdes, 1992). Note that both models predict a general impairment in responding to a previously ignored location, irrespective of the identity of the stimulus presented at that location.

An alternative explanation for the spatial negative priming effect has been proposed by Park and Kanwisher (1994). Instead of being the consequence of selecting against the irrelevant location in the prime, the so-called feature mismatching hypothesis asserts that the observed slowdown in ignored repetition trials is based on feature (e.g., shape or color) mismatches at the former prime distractor location that contains the target in the probe. For example, responding to a green probe target should be slowed down if a red distractor (but not a green distractor) had been presented at the same position during the prime. Note that the feature mismatching hypothesis is also based on the retrieval of prime information (otherwise, a mismatch could not be detected), but in contrast to the episodic retrieval model, it is assumed that there is only retrieval of perceptual prime details at the repeated location, not of the selective state during the prime ("ignore the prime distractor," "respond to the prime target"). Consequently, the feature mismatching hypothesis has nothing to do with selection against an ignored location in favor of a target and, therefore, does not predict a general impairment in responding to a previously ignored location. Instead, impaired responding is predicted only when there is a feature mismatch at the repeated location. Park and Kanwisher pitted the feature mismatching hypothesis against the distractor inhibition and the episodic retrieval models in a task based on the so-called $\mathrm{X}-\mathrm{O}$ paradigm. They presented displays containing four position markers with a target symbol (e.g., "O”) over one position marker and a distractor symbol (e.g., "X") over another. Participants had to respond to the location containing the target symbol. By exchanging the target identities between the prime (target "X," distractor " $O$ ") and the probe (target "O," distractor "X"), the probe target ("O") appeared at the same location as the prime distractor ("O") on ignored repetition trials. The feature mismatching hypothesis predicts no response slowing for this so-called match trial, because there is no prime-to-probe symbol change but a symbol match at the probe target location. The distractor inhibition model, as well as the episodic retrieval model, predicts impaired responding for match trials whenever a previously ignored location becomes the to-be-attended location. In accordance with the feature mismatching hypothesis, match trial responses were not slowed down (in fact, they were faster than responses on control trials without any location repetition).

However, considerable evidence disqualifies feature mismatching as the sole reason for visuospatial negative priming. For example, Milliken et al. (2000) showed a visuospatial negative priming effect when participants had to locate a probe target symbol " $\mathrm{X}$ " of a specific color, regardless of whether the prime distractor " $X$ " and the probe target "X" were of the same or of different colors (see also Milliken et al., 1994; Tipper et al., 1995). Here, the slowdown in responding was the aftereffect of having ignored the repeated location but was not caused by a mismatch at the repeated location. This finding can be exclusively explained by assuming distractor location inhibition or the retrieval of inconsistent prime episode 
information attached to the previous distractor location. What is more, feature mismatching is frequently regarded to be an irrelevant or, at most, a very subordinate factor in visuospatial negative priming, which is indicated by the fact that many recent visuospatial priming studies have not even excluded or controlled feature mismatching as an explanation of their spatial priming effects (Buckolz, Avramidis, \& Fitzgeorge, 2008; Buckolz et al., 2004; Fitzgeorge \& Buckolz, 2008; Gibbons, 2006; Guy \& Buckolz, 2007; Guy, Buckolz, \& Khan, 2006; Guy, Buckolz, \& Pratt, 2004). It has been assumed that a process that detects mismatching features between the current probe target and the previous prime distractor object comes into play only under very specific circumstances that have not been investigated thoroughly (Milliken et al., 1994; Tipper et al., 1995).

In contrast to visuospatial negative priming, we still know very little about auditory spatial negative priming and the underlying question of how the human cognitive system deals with locations previously occupied by irrelevant information in auditory space. There are fundamental differences between vision and audition with respect to the representation of space. Whereas the visual system is organized spatiotopically right from the retina upward, the auditory system is predominantly tonotopic. Location in auditory space is available only after being computed from interaural time and level differences, headrelated transfer functions, and distance cues (although these computations may occur as early as the superior olivary complex). This difference between modalities fits with the finding that spatial cuing effects appear to be weaker and less consistent across participants (Roberts, Summerfield, \& Hall, 2009) in the auditory, as compared with the visual, modality. Similarly, inhibition of return is smaller for auditory than for visual stimuli (Reuter-Lorenz, Jha, \& Rosenquist, 1996). Instead, there have been found precuing effects and inhibition-of-return effects with respect to sound frequency (Mondor \& Breau, 1999; Mondor, Breau, \& Milliken, 1998a; Prime \& Ward, 2002). These findings support the assumption that "space is not central to the formation of auditory objects" (Kubovy \& Van Valkenburg, 2001, p. 99). On the basis of a great amount of experimental evidence in auditory and visual perception, Kubovy and Van Valkenberg came to this conclusion when trying to specify the indispensable attributes of objecthood in audition and vision. They argued that visual objects were formed in space and time, whereas auditory objects were based in pitch and time. Following their ideas, the pitch (i.e., identity) of a sound is a fundamental/indispensable property in auditory object formation, because pitch separation, rather than location separation, is crucial for perceiving/discriminating between multiple sounds. Given these differences in the signifi- cance of space in the visual, in contrast to the auditory, modality, it is conceivable that ignoring a location in auditory space does not result in a general impairment when the same location becomes task relevant on the next trial.

Consistent with this expectation, the only auditory spatial priming study published to date revealed evidence against a general impairment in responding to a previously ignored location (Mayr, Hauke, \& Buchner, 2009b). Instead, the results supported the feature mismatching hypothesis. Participants selected a target sound on the basis of its identity while ignoring a distractor sound presented at another loudspeaker location. They reported the target's location by an appropriate keypress. Ignored repetition trials comprised a probe target sound presented at the loudspeaker location that had been occupied by the prime distractor sound. On control trials, there was no location repetition between any of the two prime and probe sounds. The pattern of results could be explained neither by inhibition of ignored locations during the prime nor by retrieval of probe-incompatible prime episode information associated with the ignored location. Instead, the data were consistent with the feature mismatching hypothesis. Negative priming (slowed-down responses to previously ignored locations, relative to control locations) occurred only for mismatch trials - that is, for trials with a sound identity change at the repeated location (which was first occupied by the prime distractor sound and then by another probe target sound). In clear contrast, responding to the previously ignored location was not slowed down on match trials on which the same sound was presented first as a prime distractor and then as a probe target at the same location. In fact, for match trials, there was an almost significant positive priming effect.

With respect to the phenomenon of auditory spatial priming, the findings by Mayr et al. (2009b) support the feature mismatching hypothesis and stand in clear contrast to the majority of studies in the visual modality. At a more general level, however, the results may be seen as indicative of object binding in the auditory modality - that is, of the combined encoding, storing, and retrieval of sound identity and location information. Otherwise - that is, if sound identity and location information had been stored and retrieved independently-the slowed-down response times for mismatch, but not for match, trials cannot be explained. A number of recent studies (Dyson \& Ishfaq, 2008; Maybery et al., 2009; Mondor, Zatorre, \& Terrio, 1998b; Parmentier, Maybery, \& Elsley, 2010; Zmigrod \& Hommel, 2009, 2010) have presented comparable evidence showing that auditory features are mandatorily integrated into auditory object files and that these object files are stored and retrieved from memory and influence subsequent performance in a similar way as 
Kahneman, Treisman, and Gibbs (1992) have proposed for the visual modality (for a review, see Hommel, 2004). ${ }^{1}$

For example, Zmigrod and Hommel (2009, 2010) presented evidence indicative of spontaneous integration of auditory features into episodic object files by showing that performance in a discrimination task was better when the second of two consecutive stimuli shared all (e.g., pitch and location) or none of the features of the first, rather than when only one of the features (either pitch or location) overlapped - that is, if a so-called partial repetition occurred. Partial repetition costs are explained by a feature integration process that establishes an episodic trace containing links between the simultaneously presented features, such as pitch and location. These episodes are assumed to be retrieved as a whole when at least one of the elements is encountered again. Retrieval does not take place when there is no repetition of any of the features. Object file retrieval is unproblematic or even beneficial for subsequent responding if all features bound together are repeated. However, partial repetitions (i.e., repetitions of some but not all features) lead to reactivation of misleading and conflicting information, which slows down processing and responding.

The findings of Mayr et al. (2009b) can be seen as another demonstration of object-binding and partial repetition costs in the auditory modality. What is new, however, is the fact that this evidence of object binding refers to a completely task-irrelevant object that should have been ignored in order to process and respond to the target stimulus. In contrast, studies of object binding are usually based on presentations of only a single task-relevant stimulus that comprises more than one feature (such as pitch and loudness, as well as pitch and location in Zmigrod \& Hommel, 2009). In these studies, it could be demonstrated that response-relevant, as well as responseirrelevant, features of the same object can be integrated into an episodic file. On the basis of the findings of Mayr, Hauke, and Buchner, however, the mechanism underlying object binding seems to be even more potent, since this mechanism is able to integrate stimulus features of an object entirely irrelevant for responding.

The findings by Mayr et al. (2009b) can be seen as evidence of partial repetition costs in auditory spatial priming and, at the same time, as evidence of the validity of the feature mismatching hypothesis. However, before this strong conclusion can be accepted as fully convincing,

\footnotetext{
${ }^{1}$ But note that interactions in auditory feature processing and binding are complex and probably heterogeneous in nature. For example, alongside evidence that argues for mandatory binding of auditory features, there is also evidence that can only be explained by an initial analysis of separate features followed by a process that integrates (binds) the separately registered features with reference to their location (Hall, Pastore, Acker, \& Huang, 2000).
}

it seems necessary to substantiate the empirical basis. Therefore, the first purpose of Experiment 1 was to replicate conceptually the Mayr, Hauke, and Buchner findings. This previous experiment had been conceptualized as the auditory equivalent of the visual $\mathrm{X}-\mathrm{O}$ paradigm, which allows for only a limited analysis of sound-identity mismatches, because there are only two sounds jointly presented on every trial throughout the experiment. This allows for a manipulation of feature matches/mismatches at the repeated (first ignored, then attended) location. This kind of mismatch directly corresponds to the mismatch concept given by Park and Kanwisher (1994). However, there is a second type of identity-location mismatch conceivable that was not implemented in the study by Mayr, Hauke, and Buchner: A mismatch can also arise when the same sound is presented as prime distractor and as probe target but its location changes. If the object-binding framework were a valid model of the mechanisms underlying auditory spatial negative priming, slowed-down responding should also be found for this second type of mismatch. We thus extended the stimulus set size from two to four environmental sounds in Experiment 1. A set of this size allows for an orthogonal manipulation of sound identity repetition and sound location repetition with respect to the prime distractor and the probe target. This allowed us to implement both mismatch types necessary to test the object-binding framework, identity changes at a repeated location and location changes of a repeated identity.

To anticipate, Experiment 1 revealed evidence compatible with the feature mismatching hypothesis in particular and the object-binding framework in general. Both types of identity-location mismatches led to slowed-down responses. In Experiment 2, we changed the experimental task from a spatial to an identity priming task but kept the auditory stimulation equivalent to that in Experiment 1 . With Experiment 2, we wanted to test whether evidence of object binding would also be found in a task that probed participants to respond to the identity of sounds (such a finding would stand in clear opposition to the previous identity negative priming literature). An additional question was whether partial repetition costs would be similar to those found in the spatial task in Experiment 1.

\section{Experiment 1}

For prime displays in Experiment 1, participants had to locate a target sound that could appear at one of four loudspeakers arranged around the participant, while ignoring another sound at one of the other three locations. In the probe display, the task was the same, but the probe target sound could appear either at the former prime distractor 
location or at a location that had not been occupied in the prime. Independently of the location repetition manipulation, the probe target could be either the same sound as that of the prime distractor or another sound that had not been presented in the prime. This two-factorial manipulation resulted in four types of trials with respect to the primedistractor-probe-target relation-that is, sound-repeated, location-repeated trials, sound-repeated, location-changed trials, sound-changed, location-repeated trials, and, finally, sound-changed, location-changed control trials in which nothing was repeated. Furthermore and on the basis of the findings in the Mayr et al. (2009b) study, we expected a pattern of results compatible with the feature mismatching hypothesis (Park \& Kanwisher, 1994), as well as the objectbinding framework - that is, slowed-down and/or more error-prone responding when the probe target sound mismatched the prime distractor sound but was presented at the same location (i.e., a sound-changed, locationrepeated trial), relative to the sound-changed, locationchanged control condition. On the basis of the objectbinding framework, we also expected to find that, as compared with the sound-changed, location-changed control condition, responding on trials with repeated sounds but changed locations (i.e., a sound-repeated, locationchanged trial) should be slowed down. In contrast, responding in the sound-repeated, location-repeated condition should not be impaired, as compared with the soundchanged, location-changed control condition, if mismatches were the sole determinant of auditory spatial negative priming; if the location and sound identity of the ignored prime distractor are repeated in the attended probe target, there can be no mismatch. If, however, distractor location inhibition or the retrieval of interfering prime episode information were also involved, responding in the sound-repeated, location-repeated condition should be impaired, relative to the sound-changed, location-changed control condition.

\section{Method}

Participants The data of 5 participants had to be eliminated due to excessive error proportions $(>.50)$ in at least one condition. Four participants did not reach the learning criterion within the maximum number of training trials. The final sample of 75 participants (62 females) ranged in age from 19 to 35 years $(M=24)$. Participants were tested individually and either were paid or received course credit.

Materials The stimuli were four digitized sounds (lamb, frog, bird, cat) of 300-ms length sampled at a rate of $44.1 \mathrm{kHz}$. The sounds differed considerably from one another with respect to spectral composition and were easily discriminable. Participants were seated in the center of a room, $60 \mathrm{~cm}$ in front of a 20 -in. iMac. The computer was connected to four Yamaha MSP3 loudspeakers. Two speakers were placed in front of the participants, one $40^{\circ}$ to the right of the sagittal head-computer-display axis, the other $40^{\circ}$ to the left. Both speakers were placed at a $1.40-\mathrm{m}$ distance from the participants. Two speakers were located in the same spatial arrangement, but behind the participants. Speakers were approximately at the height of the participants' ears.

Each trial consisted of a prime and a probe display. Each display consisted of a target presented to one location and a simultaneously presented distractor at one of the other locations. A picture appearing on the monitor before each sound display indicated the to-be-attended sound. Participants reacted to the location of the attended sound by pressing one of the four keys on the number pad of the keyboard. The key layout was spatially compatible with the locations of the loudspeakers they represented (4, front left; 5 , front right; 1 , rear left; 2 , rear right). The keys were pressed using the left and right middle and index fingers.

Prime-probe trials belonged to either the ignored repetition or the attended repetition design. In the ignored repetition design, the relation between the prime distractor and the probe target was systematically varied (as described below). In the attended repetition design, the relation between the prime target and the probe target was systematically varied. The ignored repetition design was central to the hypothesis tested, whereas the attended repetition design was primarily implemented to control for prime-to-probe response contingencies (in order to avoid predictability of probe responses based on prime responding).

For the ignored repetition design, half of the trials included a location repetition from the prime distractor to the probe target. For the other half of the trials, the location changed between the prime distractor and probe target. Sound identity was manipulated orthogonally to the location repetition manipulation. For $50 \%$ of the trials, the prime distractor sound was identical to the probe target sound; for $50 \%$ of the trials, the sound identity changed. As a consequence, $25 \%$ of the trials included a sound repetition and a location repetition between the prime distractor and probe target (sound-repeated, location-repeated), 25\% included a sound repetition but no location repetition (sound-repeated, location-changed), and 25\% included a location repetition only (sound-changed, location-repeated). For the remaining $25 \%$, there was neither a location nor a sound repetition (sound-changed, location-changed; see Fig. 1 for an illustration of the four trial types).

In a parallel way, trials in the attended repetition design belonged to one of the four sound repetition (repeated vs. changed) $\times$ location repetition (repeated vs. changed) combinations, with the only difference that the repetition 
Prime
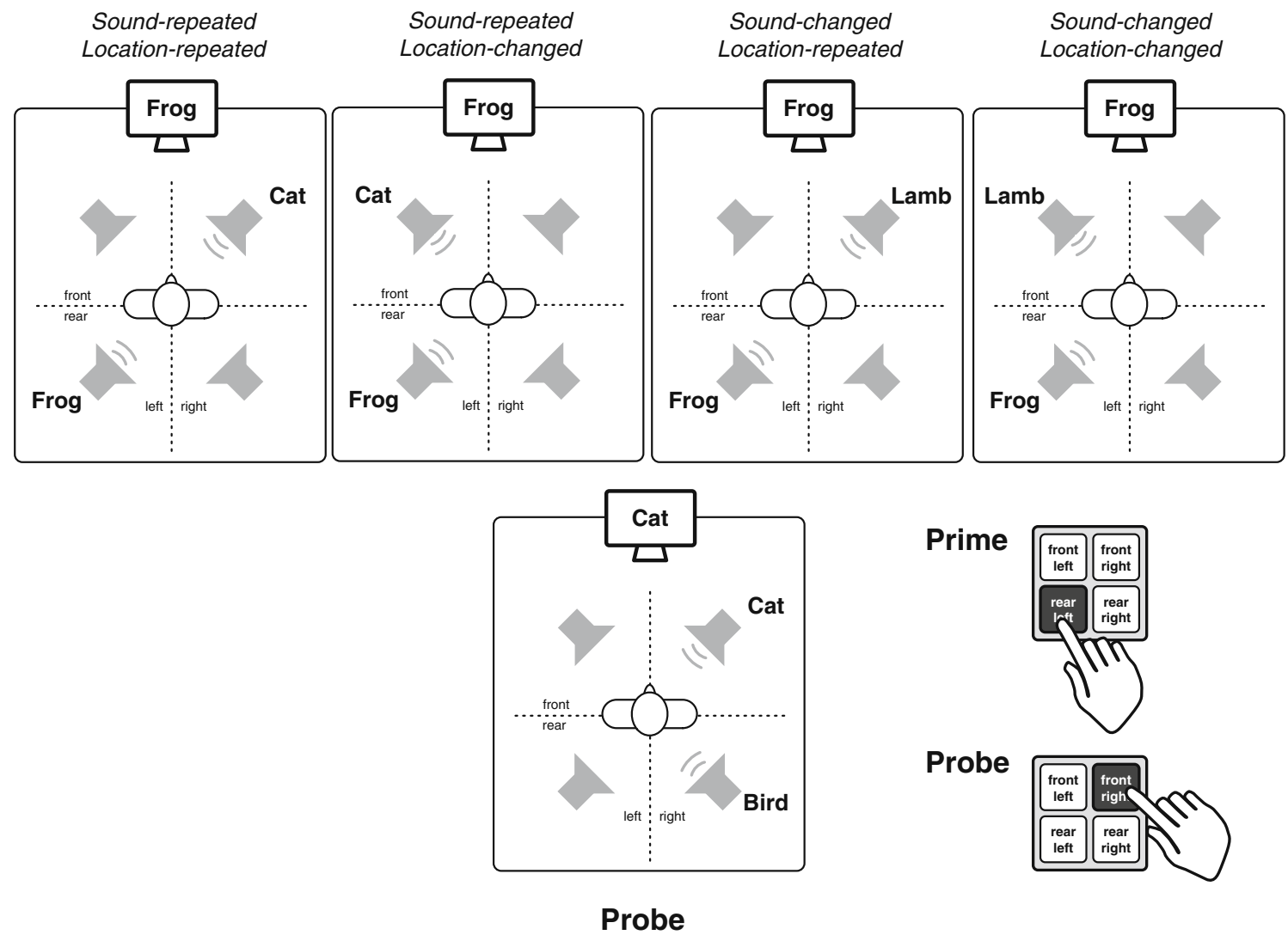

Fig. 1 Illustrated examples of the four different prime-probe trial types in the ignored repetition design in Experiment 1. The target sound was cued on the monitor. To simplify matters, the visual cue is depicted as a written word, and not in picture format (here, "frog" in

manipulation referred to the relation between the prime target and probe target. Note that $25 \%$ of the trials in each design (i.e., the sound-changed, location-changed trials) were entirely equivalent and did not include any prime-toprobe repetition; they can therefore be regarded as control trials against which the conditions with location and identity repetitions can be compared. Given the separate analyses of the two designs, we abstained from merging these two control conditions. Note, however, that additional analyses showed that response times and error rates did not differ between these two conditions when compared directly (the same was true in Experiment 2).

For a location-repeated trial in the ignored repetition design, prime and probe target and distractor locations were combined under two restrictions. The ignored prime location was identical to the attended probe location, and the attended prime location differed from the ignored probe location. For each location-repeated trial, a parallel location-changed trial was constructed by presenting the ignored prime sound at the only vacant location on the location-repeated trial. This first step resulted in a set of 24 the prime, "cat" in the probe). In the lower right of the figure, the key layout of Experiment 1 is depicted. The correct prime and probe responses for the illustrated examples are highlighted

trials, 12 location-repeated and 12 location-changed trials. This set of trials was quadruplicated.

Next, sound identity information was added. Each trial was instantiated once as a sound-repeated trial and once as a sound-changed trial. For each sound-repeated trial, the sound identities were randomly chosen, with the following restrictions. The prime distractor and probe target sound had to be identical, without any other sound repetitions. For each sound-changed trial, the sound identities were randomly chosen, with the restriction that they all had to differ from each other. In sum, the ignored repetition design comprised 192 trials, 48 in each location repetition (repeated vs. changed) $\times$ sound repetition (repeated vs. changed) condition. The construction was equivalent for the 192 trials of the attended repetition design. The 384 trials of the experiment were presented in a random sequence.

Procedure Participants first adjusted the loudness to a comfortable level. On average, the loudness was set to $65 \mathrm{~dB}(\mathrm{~A})$. Training trials were randomly chosen from the experimental trials. The experiment began only after $70 \%$ 
of the past 15 trials had been responded to correctly. If participants missed the criterion within 50 trials, they were offered the option of quitting or restarting the training.

Each experimental trial began with the visual picture cue of the sound that had to be localized subsequently. After a 500-ms interval, the two prime sounds were presented. Immediately after the prime reaction, the cue display was removed. A 1,000-ms prime-probe interval preceded onset of the visual cue for the probe. The two probe sounds were presented $500 \mathrm{~ms}$ later. Immediately after the probe reaction, the cue display was removed. In the prime as well as in the probe, reactions faster than $100 \mathrm{~ms}$ and slower than 3,000 ms were counted as invalid. Participants received audiovisual feedback about the correctness of their reactions. A 1,000-ms intertrial-interval preceded the next trial. After every 12th trial, participants received summary feedback. After the final trial, all participants were informed about the purpose of the experiment.

Design The experiment comprised a $2 \times 2$ ignored repetition design with sound repetition (repeated vs. changed) and location repetition (repeated vs. changed) as within-subjects variables and another $2 \times 2$ attended repetition design with sound repetition (repeated vs. changed) and location repetition (repeated vs. changed) as within-subjects variables. The primary dependent variable was participants' average reaction time, but error rates were also analyzed.

A power analysis was performed for the binary comparisons between the sound-changed, location-changed control trials, on the one side, and one of the other trial types, on the other. Assuming $\alpha=\beta=.05$ and a population correlation of $\rho=.3$ in the reaction times between two to-be-compared conditions, effects of size $f=0.25$ could be detected with $N=$ 75 participants (Faul, Erdfelder, Lang, \& Buchner, 2007). Whenever the statistical test of a substantive hypothesis implied a family of individual tests, the Bonferroni-Holm (Holm, 1979) method of protecting against $\alpha$-error accumulation was applied in both of the experiments reported here.

\section{Results}

Probe reaction times were evaluated only for trials with correct prime and probe responses. For the ignored repetition design (Fig. 2), the location repetition manipulation was modulated by sound repetition with trials including identity-location mismatches - that is, soundchanged, location-repeated trials and sound-repeated, location-changed trials-being slowed down, as compared with control trials without a mismatch (i.e., sound-changed, location-changed trials). In contrast, sound-repeated, location-repeated trials were not slowed down, as compared with control trials.
The family of individual tests confirmed this pattern of results. As compared with sound-changed, locationchanged control trials, sound-changed, location-repeated trials were significantly slowed down, $t(74)=3.76, p<.01$, $d_{\mathrm{z}}=0.43$, as were sound-repeated, location-changed trials, $t(74)=2.80, p=.01, d_{\mathrm{z}}=0.32$. In contrast, full repetitions (i.e., sound-repeated, location-repeated trials) did not differ from control trials (i.e., sound-changed, location-changed trials), $t(74)=0.02, p=.98, d_{\mathrm{z}}<0.01$.

The analysis of the error data did not compromise the reaction time results, since there were no significant differ-
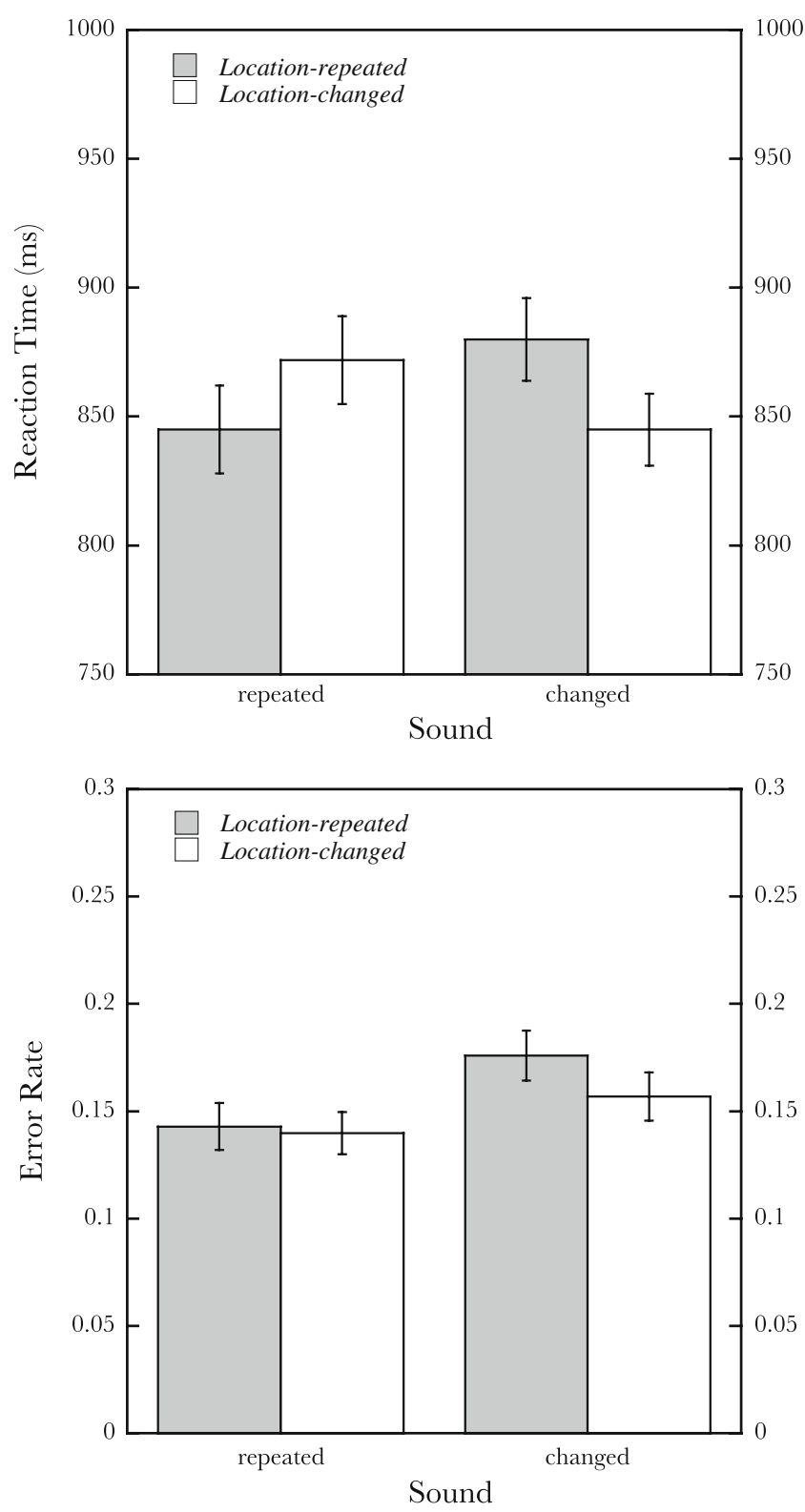

Fig. 2 Sample average of participants' mean reaction times (upper panel) and error rates (lower panel) as a function of sound repetition (repeated vs. changed) and location repetition (repeated vs. changed) for the ignored repetition design in Experiment 1. The error bars depict the standard errors of the means 
ences between any of the three comparisons, with $t(74)=$ $1.91, p=.06, d_{\mathrm{z}}=0.22$, for the comparison of sound-changed, location-repeated trials versus control trials, $t(74)=-2.00, p=$ $.05, d_{\mathrm{z}}=0.23$, for the comparison of sound-repeated, location-changed trials versus control trials, and $t(74)=$ $-1.52, p=.13, d_{\mathrm{z}}=0.18$, for the comparison of soundrepeated, location-repeated trials versus control trials.

\section{Discussion}

Experiment 1 was planned as a conceptual replication of the auditory location task reported by Mayr et al. (2009b), but with an extended stimulus set so that identity and location repetitions could be manipulated orthogonally. The present results nicely replicate and extend the findings of Mayr, Hauke, and Buchner.

First, responding was impaired, relative to the soundchanged, location-changed control condition, when there was a mismatch as originally conceived of by Park and Kanwisher (1994) — that is, when the probe target sound mismatched the prime distractor sound but was presented at the prime distractor location (i.e., a sound-changed, locationrepeated trial). Second, responding was not impaired when there was no mismatch: Responses in the sound-repeated, location-repeated condition were as fast as responses in the sound-changed, location-changed control condition. This confirms our earlier conclusion that, in auditory space, neither inhibition of ignored locations nor retrieval of incompatible prime response information attached to the ignored location affects probe responding. This is in clear contrast to the visual modality, where impaired responding to previously ignored locations has been found irrespective of the stimulus identity at the repeated location (e.g., Milliken et al., 2000).

The present findings also extend those of Mayr et al. (2009b) by showing that responding not only was slowed down when the probe target sound mismatched the prime distractor sound but was presented at the prime distractor location. Rather, responding also was slowed down when the probe target was identical to the prime distractor but appeared at a different location. In sum, then, we may conclude that auditory spatial negative priming occurs whenever there is a mismatch between prime and probe displays but does not occur when there is no mismatch, even though the probe target is a complete sound and location repetition of the prime distractor and, thus, should invite distractor inhibition, the retrieval of incompatible prime response information, or both.

Slowed-down responding in Experiment 1 entirely depended on the presence of a feature mismatch of any kind. This finding fits well within the object-binding framework. Auditory features (in a similar way as visual features) are spontaneously integrated into episodic auditory object files, which are stored in memory (Zmigrod \& Hommel, 2009,
2010). These episodes are assumed to be retrieved as a whole when at least one of the elements is encountered again on a later trial. Retrieval does not take place when there is no repetition of any of the features. This would be equivalent to our sound-changed, location-changed control trials. Object file retrieval is unproblematic or even beneficial for subsequent responding if all features bound together are repeatedthat is, in a full repetition trial. This was the case in our soundrepeated, location-repeated trials. However, partial repetitions (i.e., repetitions of some but not all features) lead to reactivation of misleading and conflicting information, which slows down processing and responding. This was true for both of our mismatch trials, the sound-changed, locationrepeated trials and the sound-repeated, location-changed trials. In sum, then, our findings argue for the validity of the object-binding framework, but with the extension that object binding occurs not only for task-relevant and task-irrelevant features of a single task-relevant object, but also for features of a totally irrelevant and interfering object that should have been ignored. Thus, the mechanisms underlying stimulus feature integration seem to be quite potent.

Similarly, at a conceptual level, but with an auditory recognition memory task, Maybery et al. (2009) presented evidence of auditory feature binding across identity and spatial information. They used sequences of auditory events that varied with respect to identity (e.g., spoken letters) and location (played from one of eight loudspeakers). A single test sound was presented from a single loudspeaker. Participants had to classify the test sound as old when it was a sound from the study sequence delivered from its original location (intact probe) or when it was a sound from the study sequence but delivered from a location that had been occupied by another study sound (recombined probe). "New" test probes comprised either a sound or a location (or both) not presented in the study sequence. If auditory identity and location information were stored separately in working memory, recognition performance for intact and recombined probes should be identical. However, it turned out that intact probes were recognized better than recombined probes, suggesting that auditory objects are stored as integrated sound identity-location representations.

The findings reported by Maybery et al. (2009) and by Zmigrod and Hommel (2009) and those of the present Experiment 1 are all demonstrations of auditory object integration of identity and spatial information. However, auditory object integration seems to be complicated by at least two aspects. First, Zmigrod and Hommel (2009) showed that features associated with task-relevant stimulus dimensions are more likely to become part of bindings than are task-irrelevant features, suggesting that attention is beneficial to the integration process. Second, there seems to be an asymmetry between the different feature dimensions in audition, in that identity information may be predomi- 
nant and may carry more weight than spatial information. For instance, Maybery et al. reported that it was easier to correctly classify as new an auditory event composed of a new sound identity and an old location than an auditory event composed of a studied sound identity at a new location. Obviously, the change of a sound's identity was more important in judging an item as new than was a change of its location. This fits well with Kubovy and Van Valkenburg's (2001) conclusion that "space is not central to the formation of auditory objects" (p. 99) but that auditory object formation is based on nonspatial properties. If the nonspatial property (such as the frequency distribution) changes, the object is no longer the same, irrespective of location repetition or change, but when the nonspatial properties remain, location changes do not impair the impression of perceiving the same object.

The asymmetry between identity and spatial information processing in auditory working memory became even more apparent when Maybery et al. (2009) slightly changed the task in their Experiments 3 and 4. Participants had to decide whether the recognition probe was an old item with respect to sound identity (verbal recognition task) or with respect to sound location (spatial recognition task). The results were task specific. Participants in the spatial recognition condition were again better at recognizing intact than recombined probes. In contrast, participants in the identity recognition condition recognized intact and recombined probes equally well. The authors concluded from this pattern of results that identity is a more fundamental dimension than spatial location in the representation of auditory objects and, as a consequence, that sound sequences could be encoded and stored with reference to their identity without including a reference to their location (Maybery et al., 2009). In contrast, it may not be possible to encode and store spatial sound information without reference to the identity of the sound. In other words, encoding sound identity information seems to be obligatory, whereas encoding location information is not. Whenever location information is central to a task, such as in the spatial recognition task, identity information is mandatorily encoded and retrieved as well, so that evidence of feature binding can be found. In contrast, when identity information is central to the task, as in the verbal recognition task for which location is irrelevant, location information is not necessarily encoded and/or retrieved, so there will be no evidence of feature binding.

Returning to the starting point of these considerationsthat is, to the auditory location negative priming task used in the present Experiment 1-location information processing was obligatory because determining the location of a sound was essential to performing the task. Following Maybery et al. (2009), obligatory auditory location processing implies encoding sound identity information as well. It suggests itself that this is why the partial repetition costs in Experiment 1 were symmetrical: Both types of mismatches - changes of the location of a repeated sound and changes of the sound identity at a repeated locationresulted in reduced performance. If these considerations are valid, the partial repetition costs may be asymmetrical when the task of locating a sound of a certain identity is replaced by the task of determining the identity of a sound at a certain location, as in an auditory identity negative priming task. Here, sound identity is central to performing the task, but location is not. Therefore, the repetition of a sound identity, but at another location, should be perceived as sound repetition with mismatching location information. In contrast, the repetition of a location occupied by another sound identity may stay undetected. In other words, for an auditory identity negative priming task, we would expect a performance decrement, relative to the sound-changed, location-changed control condition, in the sound-repeated, location-changed condition, but not in the sound-changed, location-repeated condition.

\section{Identity negative priming and feature mismatching}

While this expectation regarding performance in an identity priming task may seem reasonable within the present context, it also appears to contradict the available empirical evidence, which seems to show quite clearly that identitylocation mismatch does not play a role in auditory identity negative priming, (1) in that the size of the negative priming effect is equivalent for trials with and without a mismatch between prime and probe and (2) in that auditory negative priming can be observed in the absence of a mismatch (Buchner \& Mayr, 2004; Buchner \& Steffens, 2001; Mayr \& Buchner, 2006, 2010a, 2010b; Mayr et al., 2009a, 2010b; Mayr, Niedeggen, Buchner, \& Orgs, 2006; Mondor et al., 2005). ${ }^{2}$ How can this apparent contradiction

\footnotetext{
${ }^{2}$ Match and mismatch trials in auditory identity priming are usually implemented by manipulating whether the to-be-attended ear (indicated by a cue sound) changes between prime and probe or stays the same. For ignored repetition trials, a prime-to-probe ear change of the cue implies that the prime distractor and the probe target are presented to the same side (match trial). In contrast, if prime and probe targets are presented to the same side, ignored repetition trials are mismatch trials, because the prime distractor and the probe target are presented to different ears.

There is only one study, by Leboe et al. (2006), that has revealed evidence of feature mismatch in an auditory identity priming task (Experiment 3). However, it is questionable whether this finding investigated the processing of formerly ignored prime distractor information, since participants did not have to respond to the prime target. As a consequence, attention might not have been allocated to the prime target, but also to the prime distractor. This possibility seems to be even more plausible since there was no identity negative priming effect at all on match trials (i.e., repeated same-location trials), which is uncommon for dichotically presented identity negative priming tasks.
} 
be resolved? In short, we assume that identity-location mismatches may not have been observed in earlier auditory negative priming experiments because the sounds in those experiments had no natural location in three-dimensional space. Instead, sounds were dichotically presented over headphones, as is common practice in selective attention experiments, such that "location" was actually equivalent to the ear of entry. In such a setup, natural cues from which a sound's position in space can be computed by the auditory system either were not available at all (interaural time differences, head-related transfer functions, distance cues) or were available in a format that never occurs in real-world situations (interaural level differences: full level at one ear, zero at the other). Without any natural cues to position in space, the location feature in an auditory object file could not be computed and may thus have been missing, as a consequence of which an identity-location mismatch could not be observed.

In order to test this possibility, we decided to use an auditory identity priming task with the same fourlocation auditory free-field setup as in Experiment 1. To maximize the comparability between experiments, we also used the identical auditory stimuli, the same trial setup comprising an ignored repetition and an attended repetition design, the same response mode, and endogenous visual cues that indicated the to-be-attended location for the next display. ${ }^{3}$ On the basis of the findings in Experiment 1, the object-binding framework, and the particular significance of space in audition, we expected to find a slowdown for sound-repeated, location-changed mismatch trials, as compared with sound-changed, location-changed control trials. In contrast, on the basis of our previous findings in auditory identity negative priming, we did not expect feature mismatching to be of importance. Instead, the sound-repeated, locationchanged trials, as well as the sound-repeated, locationrepeated trials, should be slowed down, as compared with sound-changed, location-changed control trials, because the former included the repetition of a previously ignored sound identity.

\footnotetext{
$\overline{{ }^{3} \text { Experiment } 2}$ allows for a systematic investigation of feature mismatches in identity priming tasks. The dichotic presentation mode so far used in identity priming tasks did not allow us to differentiate between different types of features mismatches, as was possible in Experiment 1. In a dichotic setup, an ignored repetition trial with a feature mismatch is a trial on which the ignored prime sound switches to the other ear in the probe. However, this type of trial inextricably intermingles a location change of a particular sound identity with identity changes at specific locations (because at both ear positions, the sounds change between prime and probe). With the orthogonal manipulation of sound identity and sound location repetitions in Experiment 2, both types of mismatches can be separated.
}

\section{Experiment 2}

Method

Participants The data of 1 participant were eliminated due to excessive error frequencies $(>.50)$ in more than one condition. One further participant did not reach the learning criterion within the maximum number of training trials. The final sample of 76 participants $(61$ females) ranged in age from 16 to 40 years $(M=24.49)$. Participants were tested individually and either were paid or received course credit.

Materials and procedure The materials and procedure were identical to those in Experiment 1, with the following exceptions. Participants were seated in front of a 17-in. monitor controlled by a personal computer. The computer was connected to an APART PA 4060 amplifier controlling four JBL Control 1 Pro loudspeakers that were positioned as in Experiment 1. Participants could not adjust the loudness of the auditory presentation. Sounds were presented at about $62 \mathrm{~dB}(\mathrm{~A})$.

In Experiment 1, participants reacted to the location of the visually cued target sound. In Experiment 2, participants reacted to the identity of the sound that was presented at the visually cued target location. The visual cue was a simplified top view illustration of the participant being surrounded by the four loudspeakers (front left, front right, rear left, rear right), in which the subsequent target loudspeaker was marked by a bold red circle. As in Experiment 1, the visual cue was presented $500 \mathrm{~ms}$ before sound onset of each display and stayed on the screen until participants responded. Participants reacted to the target sound by pressing one of the four keys on the number pad of the keyboard. The response keys and the finger assignment were the same as in Experiment 1, but the sound-key association differed. Participants pressed "4" for the lamb target sound, " 5 " for the bird sound, "1" for the cat sound, and "2" for the frog sound. The keys were marked with stickers in the color that represented the particular animal in the experimental instructions (see Fig. 3 for an illustration of the four ignored repetition trial types). The identification task was somewhat more difficult than the location task in Experiment 1, because the sound-key association was not location compatible but had to be learned during the training. Therefore, the training duration was extended. Participants still had to master a criterion of $70 \%$ correct trials within the past 15 trials to enter the experiment, but the maximum number of trials was extended to 75 instead of 50 trials. If participants missed the criterion within 75 trials, they were offered the option of quitting or restarting the training. 
Prime
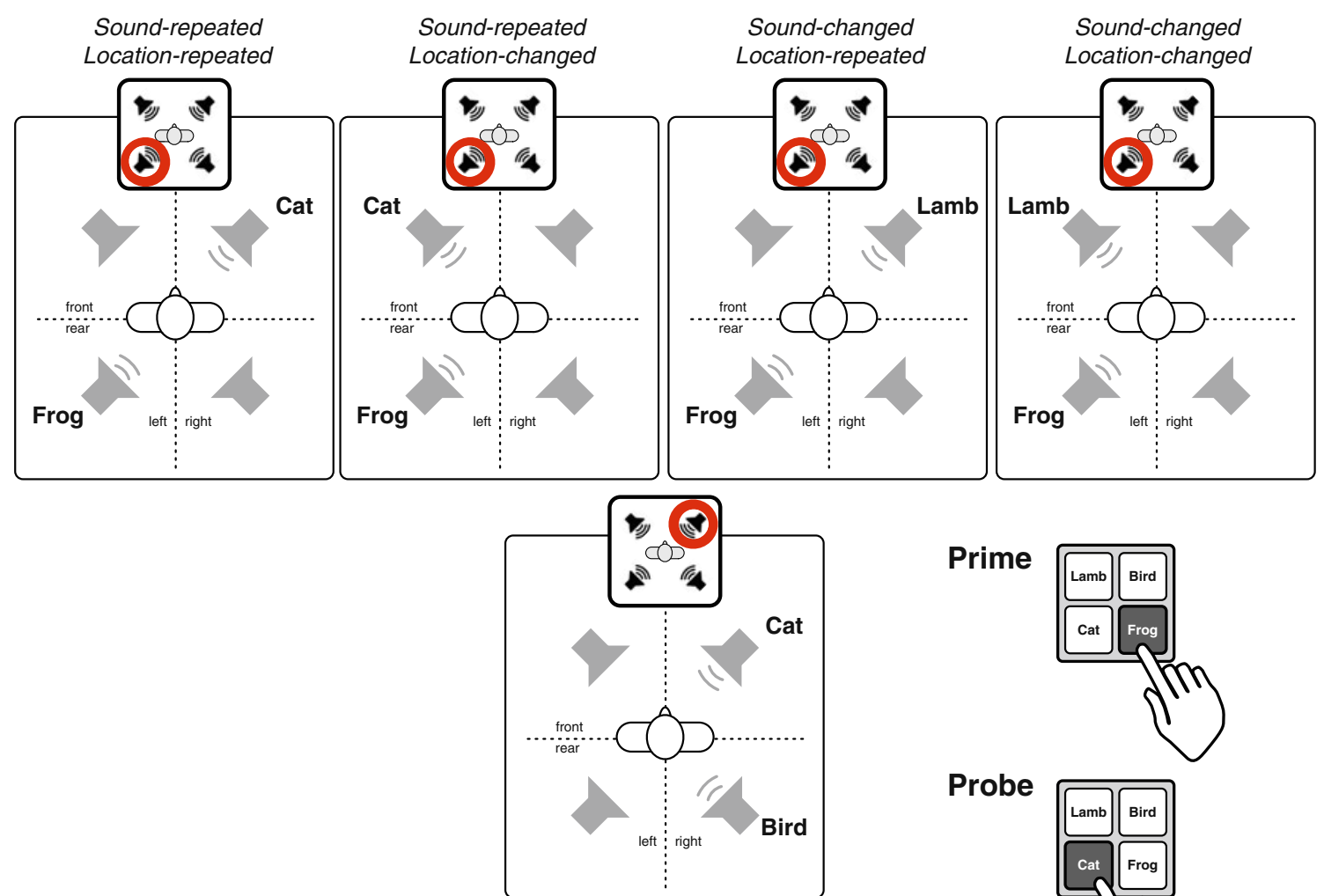

Prime
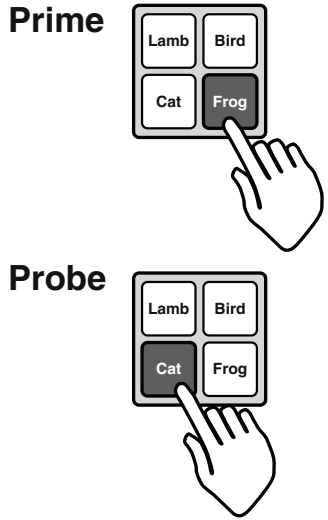

Fig. 3 Illustrated examples of the four different prime-probe trial types in the ignored repetition design in Experiment 2. The target location was cued on the monitor (here, "rear left" in the prime, "front right" in the probe). With respect to the auditory stimulation, the illustrated examples are identical to those in Fig. 1 (representing typical trials in Experiment 1).

Design The experiment comprised a $2 \times 2$ ignored repetition design with sound repetition (repeated vs. changed) and location repetition (repeated vs. changed) as within-subjects variables and another $2 \times 2$ attended repetition design with sound repetition (repeated vs. changed) and location repetition (repeated vs. changed) as within-subjects variables. The primary dependent variable was participants' average reaction time, but error rates were also analyzed.

Assuming $\alpha=\beta=.05$ and a population correlation of $\rho=.3$ in the reaction times between two to-be-compared conditions, effects with a size of $f=0.25$ could be detected with $N=76$ participants.

\section{Results}

Probe reaction times were evaluated only for trials with correct prime and probe responses. For the ignored
The difference with respect to task demands between Experiment 1 and Experiment 2 becomes apparent in the lower right, where the key layout of Experiment 2 is depicted and the correct prime and probe responses for the illustrated examples are highlighted

repetition design (Fig. 4), the location repetition manipulation was modulated by sound repetition. As compared with sound-changed, location-changed control trials, responses were slowed down when the location of a sound changed from prime distractor to probe target (i.e., in sound-repeated, location-changed trials). In contrast, there was no slowdown for trials with an identity-location mismatch as originally conceived of by Park and Kanwisher (1994) - that is, when the probe target sound mismatched the prime distractor sound but was presented at the prime distractor's location (i.e., in a sound-changed, locationrepeated trial). Similarly, full repetitions - that is, soundrepeated, location-repeated trials-were not slowed down, as compared with control trials.

The family of individual tests confirmed this pattern of results. As compared with sound-changed, locationchanged control trials, sound-repeated, location-changed trials were significantly slowed down, $t(75)=4.94, p<.01$, $d_{\mathrm{z}}=0.57$. There was a slowdown neither for sound- 

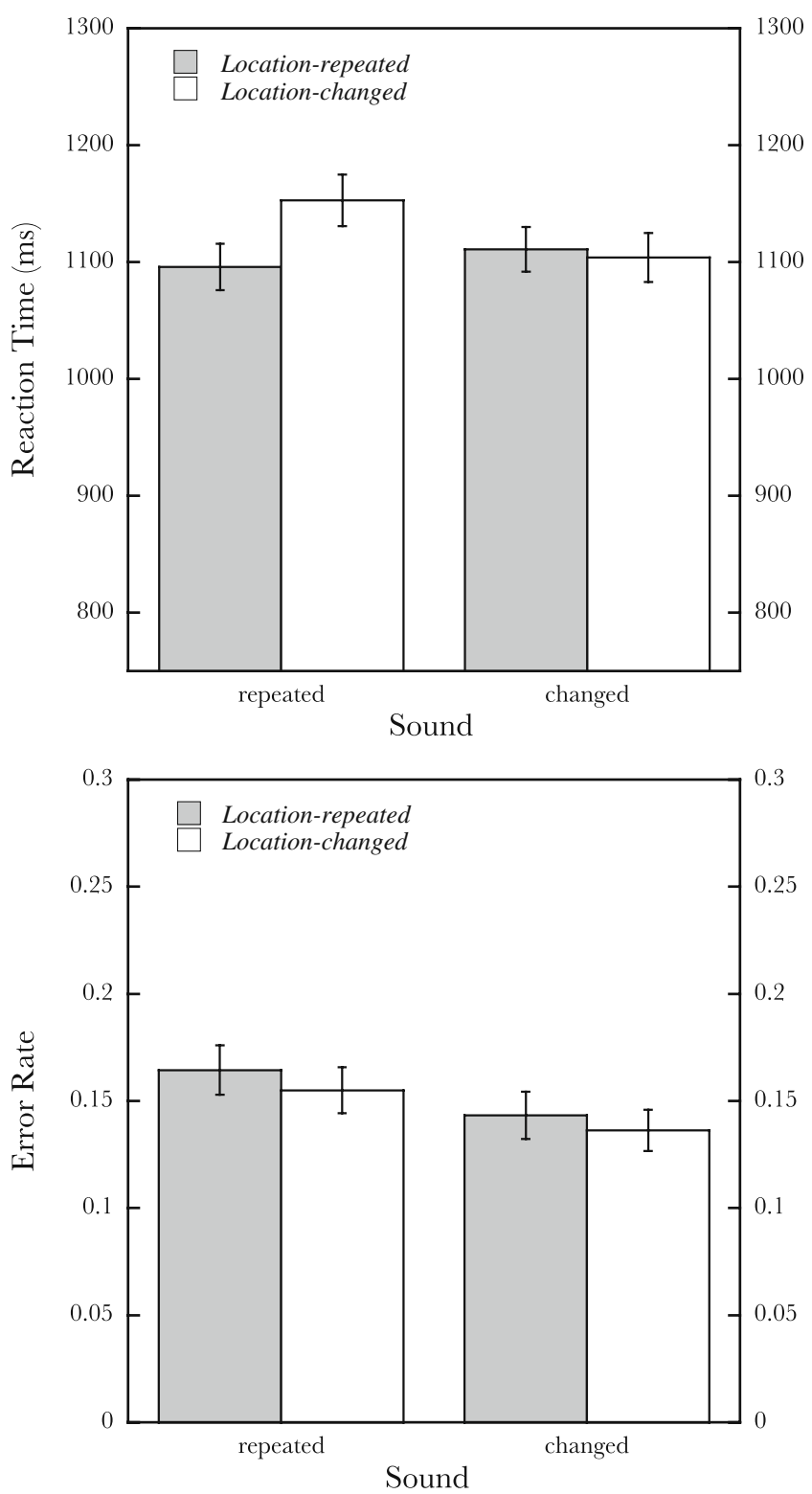

Fig. 4 Sample average of participants' mean reaction times (upper panel) and error rates (lower panel) as a function of sound repetition (repeated vs. changed) and location repetition (repeated vs. changed) for the ignored repetition design in Experiment 2. The error bars depict the standard errors of the means

changed, location-repeated trials, $t(75)=0.71, p=.48, d_{\mathrm{z}}=$ 0.08 , nor for sound-repeated, location-repeated trials, $t(75)=$ $-0.80, p=.43, d_{\mathrm{z}}=0.09$.

The error data did not contradict the reaction time data. The sound-repeated, location-changed trials did not differ from the sound-changed, location-changed control trials, although the error rate for this type of mismatch trial was almost significantly larger, $t(75)=2.16, p=.03, d_{\mathrm{z}}=0.25$. There was no difference between the sound-changed, location-repeated trials and the control trials, $t(75)=0.69$, $p=.50, d_{\mathrm{z}}=0.08$. The sound-repeated, location-repeated full repetition trials were the only trials with increased error rates, as compared with the control trials, $t(75)=3.16, p<$ $.01, d_{\mathrm{z}}=0.36$.

\section{Discussion}

Experiment 2 was planned to test whether an identity negative priming task would reveal evidence of identitylocation mismatches if the auditory stimulation was identical to that of the spatial negative priming task in Experiment 1 and, if so, whether the partial repetition costs were symmetric or not. Indeed, participants were impaired in responding to the identity of a previously ignored stimulus when the same stimulus had been presented at another location in the prime. Impaired responding in mismatch trials of the sound-repeated, location-changed type was in accordance with the results of Experiment 1, in which participants had to localize target stimuli. Obviously, identity-location mismatches can modulate negative priming effects in auditory identity priming tasks. However, this finding is in contrast with previously reported auditory identity negative priming studies in which a location change of the prime distractor did not modulate the size of the negative priming effect (Buchner \& Mayr, 2004; Buchner \& Steffens, 2001; Mondor et al., 2005; but see Leboe et al., 2006). The most likely explanation for this discrepancy between earlier auditory negative priming experiments and Experiment 2 seems to be that in the former experiments, there were no natural cues to position in three-dimensional space, due to the dichotic presentation mode over headphones. Without any natural localization cues, the location feature in an auditory object file could not be computed and may, thus, have been missing, as a consequence of which an identity-location mismatch could not be observed.

Furthermore, and in contrast to Experiment 1, Experiment 2 showed no evidence of partial repetition costs to mismatches as originally conceived by Park and Kanwisher (1994) - that is, when the sound at the repeated location changed between prime and probe (in sound-changed, location-repeated trials). The asymmetric pattern of partial repetition costs as evidenced here-a performance decrement in the sound-repeated, location-changed condition, but not in the sound-changed, location-repeated condition-is in line with the findings of Maybery et al. (2009) and in accordance with their assumption that location information is not necessarily encoded or retrieved when identity information is central to the task. As a consequence, the identity-location mismatch in a sound-changed, locationrepeated trial is not detected and, thus, does not impair responding.

There is one additional detail of Experiment 2 that should be mentioned here. As we have argued above, the 
detection of identity-location mismatches resulting in slowed-down responding has probably been due to the way the auditory stimulation had been implemented in Experiment 2, but not in earlier experiments. However, one discrepancy between the results of Experiment 2 and those of earlier identity negative priming experiments still needs to be addressed: Why should the auditory three-dimensional spatial layout of Experiment 2 prevent the appearance of any identity negative priming effect beyond the detection of identity-location mismatches? If anything, we would have expected a reduced negative priming effect (but not the complete absence of negative priming) for sound-repeated, location-repeated trials without an identity-location mismatch due to processes such as distractor inhibition (Tipper, 1985, 2001; Tipper $\&$ Cranston, 1985) or the retrieval of incompatible prime response information (Mayr \& Buchner, 2006; Neill \& Valdes, 1992; Neill et al., 1992; Rothermund et al., 2005) usually involved in identity priming tasks. This discrepancy is more apparent than real, as is revealed by taking a closer look at the error data in Experiment 2, which indicate the involvement of processes that go beyond the sole detection of identity-location mismatches. For both trial types with a sound repetition - that is, for the soundrepeated, location-repeated trials and for the soundrepeated, location-changed trials-there was an increase in error rates, as compared with the trials without a sound repetition, although this increase in error rates, as compared with control trials, was significant only for sound-repeated, location-repeated trials. In fact, an supplementary $2 \times 2$ (sound repetition $\times$ location repetition) MANOVA of the error rates revealed a significant main effect of sound repetition, $F(1,75)=$ $9.45, p<.01, \eta^{2}=.11$. The main effect of location repetition and the interaction between both variables were not significant, $F(1,75)=1.24, p=.27, \eta^{2}=.02$, and $F(1,75)=0.04, p=.85, \eta^{2}<.01$, respectively. In other words, irrespective of location change or repetition, when the sound was repeated from prime distractor to probe target, there was an increase in response errors. This increase in errors due to sound repetition is probably the correlate of a small identity negative priming effect that might have resulted from distractor identity inhibition or the retrieval of incompatible prime response information. Furthermore, this increase in errors to repeated sound identities was evidenced only in the identification task in Experiment 2, but not in the spatial task in Experiment 1. This seems to indicate that in addition to matching processes, distractor inhibition or the retrieval of incompatible prime response information is involved, but only when identity information is response relevant, and not when spatial information is response relevant.

\section{Attended repetition design: Evidence of stimulus $\times$ response repetition effects}

The ignored repetition design was central to the hypothesis tested, whereas the attended repetition design had been implemented to control for prime-to-probe response contingencies and, thus, to prevent the development of response strategies such as "never repeat a prime response." We generally abstain — and do so here as well-from directly comparing ignored repetition with attended repetition trials, because such a comparison confounds at least two differences. First, the two types of repetition trials differ in that attention is allocated to a location that either was in the focus of attention previously (in attended repetition trials) or was ignored due to its task irrelevance on the previous trial (in ignored repetition trials). Second, on attended repetition trials, participants have to respond to the repeated location that contains the prime target. As a consequence, differences between attended repetition and ignored repetition trials may be caused not only at an early processing stage (attending vs. ignoring the repeated location), but also at a later stage (responding vs. not responding to the repeated location). In accordance with the bypass rule (Fletcher \& Rabbitt, 1978; Krueger \& Shapiro, 1981), participants are inclined to repeat a response if the stimuli are the same across adjacent trials (due to the formation of a direct link from early stimulus representation to the response; see Pashler \& Baylis, 1991), but they will tend to change the response if the stimuli differ. Consequently, the origin of our attended repetition effects (perceptual due to location repetition and/or postperceptual due to the bypass rule) stays ambiguous. Nevertheless the results of the attended repetition design are informative in that they shed some light on auditory stimulus $\times$ location repetition effects.

\section{Experiment 1}

In the attended repetition design in Experiment 1 (Fig. 5, left side), the sound repetition manipulation was modulated by location repetition. In the $2 \times 2$ MANOVA of the reaction time data with sound repetition (repeated vs. changed) and location repetition (repeated vs. changed) as within-subjects variables, the main effects of sound repetition and location repetition were statistically significant, $F(1,74)=293.78$, $p<.01, \eta^{2}=.80$, and $F(1,74)=23.62, p<.01, \eta^{2}=.24$, respectively. The interaction between both variables was also significant, $F(1,74)=134.18, p<.01, \eta^{2}=.65$. For trials with a sound repetition between prime target and probe target, responding was facilitated when there was an additional location repetition, $t(74)=11.70, p<.01, d_{\mathrm{z}}=$ 1.35. In contrast, for trials with a sound change, location repetition responding was particularly slow, $t(74)=5.43, p<$ $.01, d_{\mathrm{z}}=0.63$. 

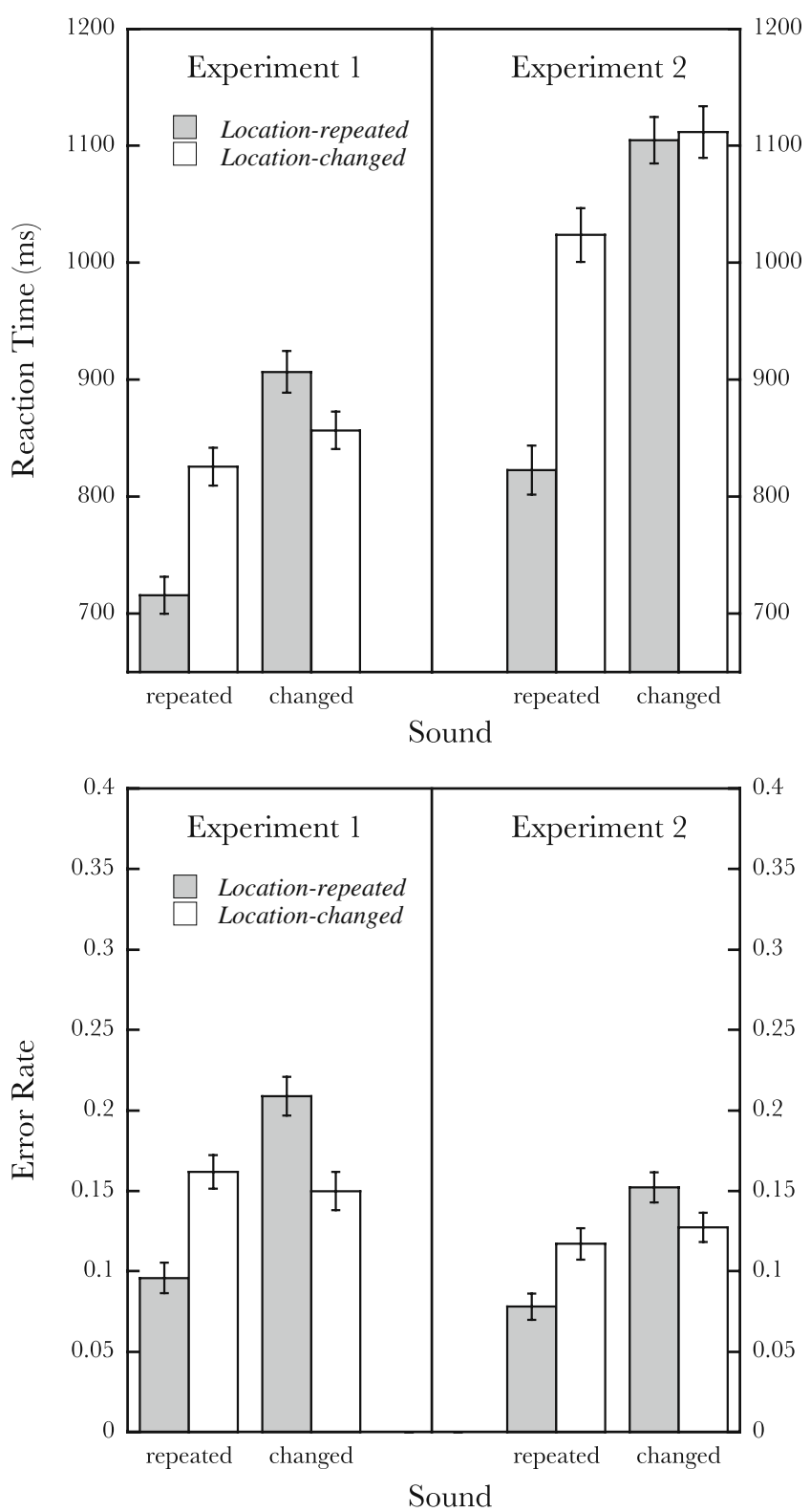

Fig. 5 Sample average of participants' mean reaction times (upper panel) and error rates (lower panel) as a function of experiment (Experiment 1 vs. Experiment 2), sound repetition (repeated vs. changed), and location repetition (repeated vs. changed) for the attended repetition designs in both experiments. The error bars depict the standard errors of the means

An analysis of the error data revealed a significant main effect of sound repetition, $F(1,74)=64.30, p<.01, \eta^{2}=.47$. The main effect of location repetition was not significant, $F(1,74)<0.25, p=.62, \eta^{2}<.01$. The interaction between both variables was significant, $F(1,74)=62.45, p<.01$, $\eta^{2}=.46$. In accordance with the reaction time data, error rates decreased for trials with a sound repetition from prime to probe target when the location was also repeated, $t(74)=$ $6.06, p<.01, d_{\mathrm{z}}=0.70$. For trials with a sound change, there was an error increase when the location was repeated, $t(74)=$ $5.27, p<.01, d_{\mathrm{z}}=0.61$.
Experiment 2

For the attended repetition design in Experiment 2 (Fig. 5, right side), the sound repetition manipulation was modulated by location repetition. In the $2 \times 2$ MANOVA of the reaction time data, with sound repetition (repeated vs. changed) and location repetition (repeated vs. changed) as within-subjects variables, the main effects of sound repetition and location repetition were statistically significant, $F(1,75)=237.68, p<.01, \eta^{2}=.76$, and $F(1,75)=112.10$, $p<.01, \eta^{2}=.60$, respectively. The interaction between both variables was also significant, $F(1,75)=160.17, p<.01$, $\eta^{2}=.68$. Responding was facilitated for trials with a sound repetition when the location was also repeated between prime target and probe target, $t(75)=14.21, p<.01, d_{\mathrm{z}}=$ 1.63. In contrast, when the sound changed, there was no difference between trials with a location repetition and a location change, $t(75)=0.61, p=.54, d_{\mathrm{z}}=.07$.

$\mathrm{An}$ analysis of the error data revealed a significant main effect of sound repetition, $F(1,75)=42.48, p<.01$, $\eta^{2}=.36$. The main effect of location repetition was not significant, $F(1,75)=1.13, p=.29, \eta^{2}=.02$. The interaction between both variables was significant, $F(1,75)=28.61, p<.01, \eta^{2}=.28$. For trials with a sound repetition between prime target and probe target, there was a decrease in error rates when the location was repeated as well, $t(75)=4.36, p<.01, d_{\mathrm{z}}=.50$. For trials with a sound change, there was an increase in errors when the location was repeated, $t(75)=2.76, p=.01, d_{\mathrm{z}}=.32$.

The following conclusions can be drawn from the attended repetition design results of Experiment 1 and 2 . Overall, the results are characterized by strong interaction effects between the sound repetition and location repetition variables. Irrespective of whether participants accomplished a location (Experiment 1) or an identity (Experiment 2) task, responding was tremendously facilitated (in comparison with all the other experimental conditions) when identical target sounds were presented at the same target location in the prime and probe. In addition, responding was particularly slowed down, as compared with soundchanged, location-changed control trials, when the target sound changed identity between the prime and probe but was presented at the same location (in sound-changed, location-repeated trials). This type of partial repetition effect was larger - both in reaction times and in error ratesin the location task (Experiment 1) than in the identification task (Experiment 2). In the identification task, there was only a significant increase in error rates for trials with a sound change at the repeated location, but there was no slowdown in reaction times. In sum, responding was clearly facilitated for full sound and location repetitions, and responding was impaired when partial repetitions in the sound-changed, location-repeated trials occurred. 
The figures clearly show that the full-/partial repetition effects were much stronger in the attended repetition than in the ignored repetition design. ${ }^{4}$ This can be due to two reasons, as already noted above. First, repeated attention to a location or a sound identity may increase the effect of a (mis)match detection. Second, the detection of a (mis) match at a repeatedly attended location interferes not only at the level of perceptual processing, but also at a later processing stage. In accordance with the bypass rule (Fletcher \& Rabbitt, 1978; Krueger \& Shapiro, 1981), participants are usually inclined to repeat a response if the stimuli are the same across adjacent trials. This is what most likely happened in trials with a full repetition of sound identity and location, since in both tasks, a full repetition came along with a response repetition requirement. The bypass rule further leads to the prediction that participants will tend to change their response if the stimuli differ. Consequently, response repetitions, despite a stimulus identity change, should be particularly difficult. This explains why participants were markedly slowed down on sound-changed, location-repeated trials in the spatial task (Experiment 1) when the attended stimulus changed identity but the location was repeated and, therefore, the response had to be repeated. Responding on the same sound-changed, location-repeated trials was less strongly impaired in the identification task (Experiment 2), possibly because, in this task, these trials did not require a response repetition between prime and probe.

\section{A multinomial model analysis of error types: Evidence of prime response retrieval processes}

To provide additional support for the involvement of processes beyond the detection of identity-location mismatches, we ran a supplementary analysis. We know from a number of our earlier identity priming experiments that participants have a tendency to erroneously repeat the former prime response in ignored repetition, as compared with control, trials (Mayr \& Buchner, 2006, 2010a; Mayr et al., 2009a; Mayr, Möller, \& Buchner, in press). This pattern of results has been interpreted as evidence of prime response retrieval processes (for a similar account, see also Rothermund et al., 2005). The probe target on an ignored repetition trial may serve as a retrieval cue for the whole prime episode, including the prime target and the prime

\footnotetext{
$\overline{{ }^{4} \text { According to }}$ this observation, for both experiments, a $2 \times 2 \times 2$ MANOVA including the factor of subdesign (attended repetition vs. ignored repetition), in addition to the factors identity repetition (repeated vs. changed) and location repetition (repeated vs. changed), revealed significant three-way interactions, for both reaction times and error rates.
}

response. In contrast, there is no stimulus repetition from prime to probe on control trials and, consequently, no retrieval of prime episode, prime target, and prime response information takes place. Furthermore, the auditory localization task used by Mayr et al. (2009b) showed no evidence of prime response retrieval effects on trials with a prime distractor-to-probe target location repetition, presumably because action-oriented episodes are not stored (and thus cannot be retrieved) in auditory location-priming paradigms.

In order to test whether there was evidence of prime response retrieval in identity priming (i.e., of an increase in prime response errors on the trials with a sound identity repetition, in comparison with trials without a sound repetition), we ran an additional analysis on the categorical response data in Experiment 2, using an appropriate multinomial model as in our earlier experiments. In a similar way, we analyzed the categorical response data in Experiment 1 and expected to replicate the absence of a prime response retrieval effect in this localization task.

The error frequency data underlying the multinomial analyses are displayed in Table 1. We used the multinomial model introduced by Mayr and Buchner (2006, for details) but adapted it to the experimental design of the present Experiment 1 and 2. Instead of two processing trees in a standard negative priming task-one for the ignored repetition condition and one for the control condition - we designed one processing tree for each of the four possible combinations of sound repetition (repeated vs. changed) and location repetition (repeated vs. changed). The model implies that participants correctly identify and respond to the probe target (location in Experiment 1, identity in Experiment 2) with probability $c i$. Errors $(1-c i)$ may have different causes. Participants may confuse the probe target and distractor and respond with the probe distractor (identity or location) with conditional probability $p s c$. If probe stimulus confusion does not dominate responding $(1-p s c)$, then, with probability $p r r$, prime response retrieval may occur and lead to incorrect prime response repetitions. Parameter prr thus is critical for the present purposes.

The estimates of the four prr parameters for Experiment 1 and 2 are illustrated in the upper and lower panels of Fig. 6, respectively. For the spatial task in Experiment 1, we did not expect prime response retrieval to be increased in any of the four trial type conditions. Therefore, the probability of retrieving a prime response, prr, was expected to be equivalent for all four trial types. In other words, for Experiment 1, the empirical data should fit the restricted model assuming $p r r_{\text {sound-repeated, location-repeated }}=p r r_{\text {sound- }}$ repeated, location-changed $=p r r_{\text {sound-changed, location-repeated }}=$

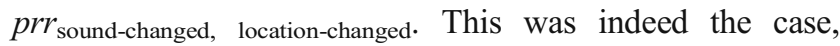


Table 1 Accumulated absolute frequencies of correct probe responses and of the different types of probe errors as a function of sound repetition (repeated vs. changed) and location repetition (repeated vs. changed) for the ignored repetition design in Experiment 1 (above) and Experiment 2 (below)

\begin{tabular}{|c|c|c|c|c|}
\hline & \multicolumn{4}{|l|}{ Experiment $1(N=75)$} \\
\hline & $\begin{array}{l}\text { Sound-repeated, location- } \\
\text { repeated }\end{array}$ & $\begin{array}{l}\text { Sound-repeated, location- } \\
\text { changed }\end{array}$ & $\begin{array}{l}\text { Sound-changed, location- } \\
\text { repeated }\end{array}$ & $\begin{array}{l}\text { Sound-changed, location- } \\
\text { changed }\end{array}$ \\
\hline $\begin{array}{l}\text { Correct probe target } \\
\text { responses }\end{array}$ & 2,611 & 2,590 & 2,474 & 2,579 \\
\hline $\begin{array}{l}\text { Incorrect probe distractor } \\
\text { responses }\end{array}$ & 209 & 181 & 271 & 231 \\
\hline $\begin{array}{l}\text { Incorrect prime target } \\
\text { responses }\end{array}$ & 69 & 76 & 85 & 100 \\
\hline \multirow[t]{2}{*}{ Other incorrect responses } & 127 & 141 & 147 & 122 \\
\hline & \multicolumn{4}{|l|}{ Experiment $2(N=76)$} \\
\hline $\begin{array}{l}\text { Correct probe target } \\
\text { responses }\end{array}$ & 2,630 & 2,633 & 2,697 & 2,698 \\
\hline $\begin{array}{l}\text { Incorrect probe distractor } \\
\text { responses }\end{array}$ & 386 & 383 & 369 & 351 \\
\hline $\begin{array}{l}\text { Incorrect prime target } \\
\text { responses }\end{array}$ & 64 & 53 & 21 & 22 \\
\hline Other incorrect responses & 37 & 29 & 41 & 36 \\
\hline
\end{tabular}

$G^{2}(3)=6.25, p=.10 .^{5}$ As a consequence, the model with the restriction that all four prr probabilities are equal cannot be rejected.

For Experiment 2, in contrast, the goodness-of-fit test of the restricted model assuming the equality of all four prr parameters was expected to lead to a significant misfit, because we expected the probability of retrieving a prime response to be larger on the sound-repeated, locationrepeated trials, as well as on the sound-repeated, locationchanged trials, than in the two trial types without a sound repetition. This misfit was indeed observed, $G^{2}(3)=23.46$, $p<.01$, and thus, the model had to be rejected. However, the data should fit a model with equal prr parameters on trials with a sound repetition ( $p r r_{\text {sound-repeated, location-repeated }}=$ $p r r_{\text {sound-repeated, location-changed }}$ ) and on trials without a soundrepetition ( $p r r_{\text {sound-changed, location-repeated }}=p r r_{\text {sound-changed, }}$ location-changed). This was indeed the case, $G^{2}(2)=0.25$, $p=.88$.

The supplementary multinomial analyses revealed that prime response retrieval processes, as indicated by an increase of prime response errors, were involved in the identification task in Experiment 2, but not in the localization task in Experiment 1. When a previously ignored sound identity became response relevant in Experiment 2, as was the case on sound-repeated,

\footnotetext{
${ }^{5}$ The log-likelihood goodness-of-fit statistic $G^{2}$ is asymptotically $\chi^{2}$ distributed, with the degrees of freedom indicated in parentheses (see $\mathrm{Hu} \&$ Batchelder, 1994, for details). The goodness-of-fit test was conducted using the AppleTree program (see Rothkegel, 1999).
}

location-repeated and sound-repeated, location-changed trials, the probability of erroneously repeating the former prime response increased. Whether there was a prime distractor-to-probe target location repetition or not did not make any difference with respect to this specific error type. This implies that only sound repetitions, but not location repetitions, can cue the retrieval of prime response information. This is in accordance with the overall pattern of Experiment 2, which revealed that only sound repetitions, but not location repetitions, functioned as successful retrieval cues for the prime. This finding is also in accordance with a number of previous identity priming studies that have revealed an increase in prime response retrieval errors on ignored repetition trials, as compared with control trials (Mayr \& Buchner, 2006, 2010a; Mayr et al., 2009a, in press).

For the localization task in Experiment 1, however, the probability of prime response retrieval errors was equivalent across all trial types. Neither the repetition of locations nor the repetition of identities resulted in an increase of prime response errors. This result replicates the findings reported by Mayr et al. (2009b), who did not find any evidence of the involvement of prime response retrieval processes in auditory spatial priming. In combination with the findings from the main analysis, Experiment 1 revealed clear evidence of object file retrieval, as indicated by the partial repetitions costs, but no evidence of retrieved response information. This allows us to conclude that action-oriented episodes may not be stored in the case of auditory location priming. While this 

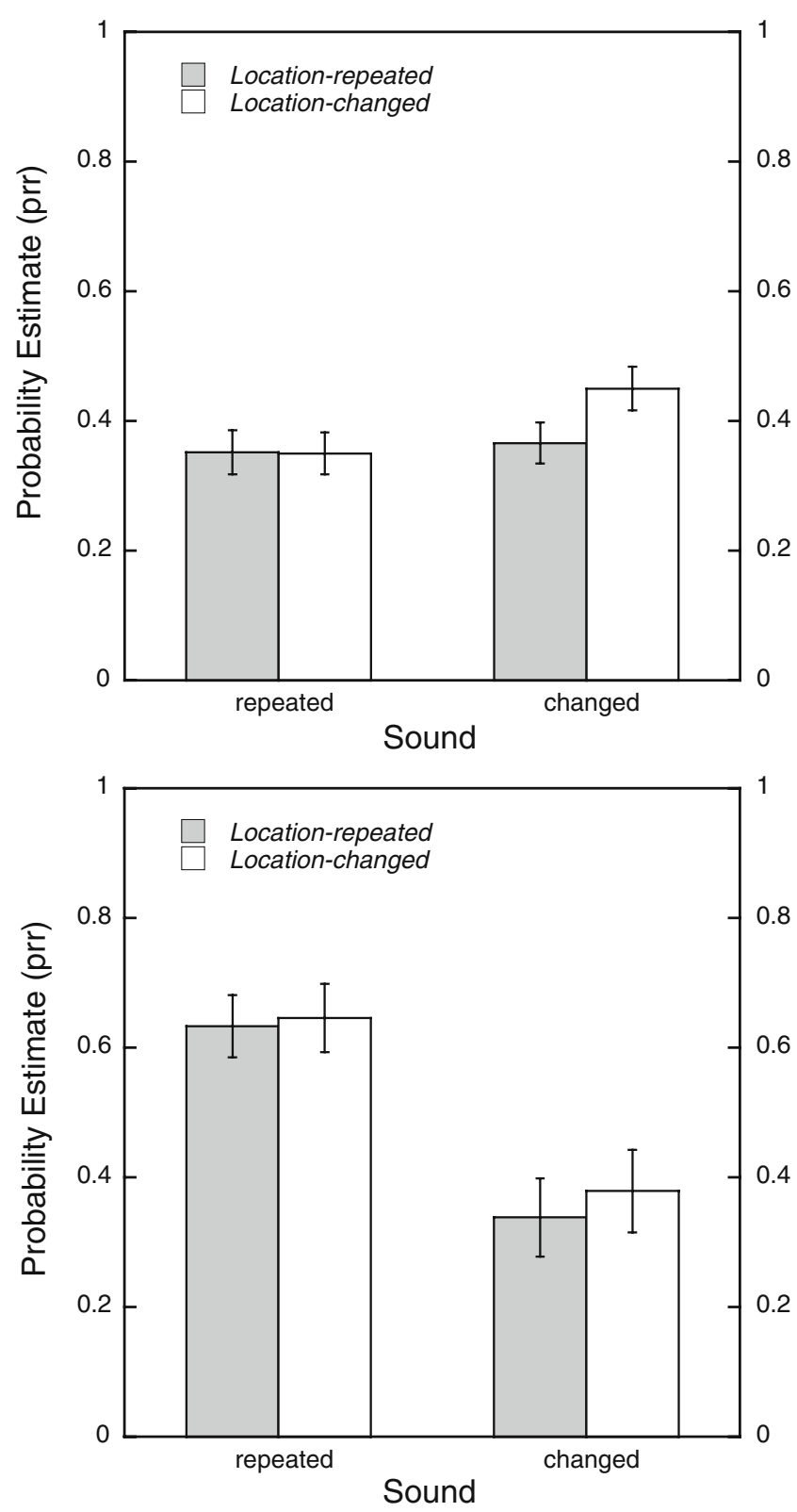

Fig. 6 Probability estimates of the model parameter estimating the probability of prime response retrieval as a function of sound repetition (repeated vs. changed) and location repetition (repeated vs. changed) for the ignored repetition design in Experiment 1 (above) and Experiment 2 (below). The error bars depict the standard errors of the means

conclusion might stand to reason, given the replicated findings, an explanation of why spatial negative priming does not involve the generation and storage of actioncentered episodes is less obvious. What is specific to responding in the spatial task is the fact that responses are related to locations, irrespective of what kind of identity is presented at this location. In other words, a specific response in a localization task is independent of the target sound's identity. This suggests that prime response information can be retrieved (and, thus, cause errors) only via retrieval of the prime target identity, which, in turn, is part of the cued prime episode. This would mean that response information is usually closely attached to the memory representation of the prime target identity. If, however, there is no fixed target identity-response relation, as in localization tasks, no target identity-response binding might be generated. As a consequence, retrieval of prime episodes in a localization task does not include retrieval of prime response information.

\section{General discussion}

Here we presented two negative priming experiments based on an identical auditory setup. A spatial negative priming task requiring localization responses to a cued sound identity was used in Experiment 1. An identity negative priming task requiring identification responses to a sound at a cued location was used in Experiment 2.

Experiment 1 revealed evidence of identity-location mismatches and, thereby, nicely replicated and extended the findings of Mayr et al. (2009b). First, responding was impaired, relative to the sound-changed, location-changed control condition, when there was a mismatch as originally conceived of by Park and Kanwisher (1994) - that is, when the probe target sound mismatched the prime distractor sound but was presented at the prime distractor's location (i.e., a sound-changed, location-repeated trial). Second, responding was not impaired when there was no mismatch: Responses in the sound-repeated, location-repeated condition were as fast as responses in the sound-changed, location-changed control condition. Experiment 1 also extended the findings of Mayr et al. by showing that responding was slowed down when the probe target was identical to the prime distractor but appeared at a different location. In other words, auditory spatial negative priming occurs whenever there is a mismatch between prime and probe displays but does not occur when there is no mismatch, even though the probe target is a complete sound and location repetition of the prime distractor and, thus, should invite distractor inhibition or the retrieval of incompatible prime response information. On the basis of our findings, spatial selection processes in the auditory modality seem to work quite differently than visuospatial selection processes. While the former do not reveal any evidence of a general impairment in responding to previously ignored locations, the latter comprise a spatial inhibition component for task-irrelevant locations (see, e.g., Buckolz et al., 2004; Chao, 2009; Milliken et al., 1994, 2000; Neill et al., 1992, 1994; Tipper et al., 1990, 1995). Obviously, ignored locations in auditory space are neither marked as irrelevant, in the sense of a do-not-respond tag 
(Neill et al., 1992), nor inhibited in order to enable efficient responding to the target location (Tipper et al., 1995). Buckolz and colleagues (Buckolz et al., 2008; Buckolz et al., 2004) have shown that part of the visuospatial negative priming effect is based on response inhibition to the prime distractor location. It is assumed that the (motor) response to the prime distractor location is inhibited in order to facilitate prime target responding. As a consequence, leftover response inhibition impairs responding to the prime distractor location when it becomes the target location in the probe. In the visual modality, the necessity of such a response inhibition mechanism applied to distractor locations seems to be plausible, since there is evidence that subliminally presented (masked) prime stimuli can have strong automatic influences on motor activation (see, e.g., Eimer \& Schlaghecken, 1998). To our knowledge, there are no comparable automatic effects of sound presentation on motor activation. Therefore, a response inhibition mechanism that prevents inadequate motor responses to to-beignored sounds at distractor locations might not be necessary.

The relevance of identity-location mismatches that was found in both of our experiments is indicative of the importance of object binding in auditory spatial negative priming - that is, of the combined encoding, storing, and retrieval of sound identity and location information. If one of the features changes but the other repeats, partial repetition costs are observed. As such, Experiment 1 ties in with a number of studies that have reported evidence of binding into object files in auditory short-term memory (Dyson \& Ishfaq, 2008; Maybery et al., 2009; Mondor et al., 1998b; Parmentier et al., 2010; Zmigrod \& Hommel, 2009, 2010).

In contrast to the multitude of previous auditory identity negative priming experiments (Banks et al., 1995; Buchner \& Mayr, 2004; Buchner \& Steffens, 2001; Mondor et al., 2005), there was also evidence of identity-location mismatches in identity negative priming in Experiment 2. Negative priming was present when the ignored sound identity changed location in the probe (on sound-repeated, location-changed trials), when compared with soundchanged, location-changed control trials. In contrast, without an identity-location mismatch when the same sound was repeated at the same location (on soundrepeated, location-repeated trials), there was no slowdown in responding. This pattern of results clearly argues for the relevance of identity-location mismatches in identity negative priming.

The most plausible explanation for the different importance of identity-location mismatches in Experiment 2, as compared with previous auditory identity negative priming experiments, is the way location in auditory space was represented. In all previous auditory negative priming experiments, sounds were presented over headphones, such that "location" was equivalent to the ear of entry but natural localization cues were either missing or not available in a realistic format. We assume that in the absence of natural localization cues, the location feature in an auditory object file could not be computed. Missing location information in a retrieved object file rules out the detection of identity-location mismatches. ${ }^{6}$ Our finding is not the only evidence that the naturalness of localization cues might be of particular importance in auditory attention tasks with a spatial component. In a recent study pursuing the question of whether covert spatial-orienting benefits in a nonspatial auditory task depend on the spatial relevance of the cues, Roberts et al. (2009) compared the benefits of informative cues presented in a free-field environment with three different headphone presentation conditions - that is, (1) a virtual space condition generated by head-related transfer functions, (2) a binaural condition containing interaural time differences only, and (3) a binaural condition containing interaural-level differences only. The free-field condition was the only condition in which participants gained significant benefits from valid spatial cues both with respect to reaction times as well as error rates. Possibly, the authenticity in representing space is all the more of importance in a negative priming task where the location processing of a distractor sound takes place while target sound processing is in the focus of attention. As a consequence of the present findings and of those reported by Roberts et al. (2009), we can only recommend making sure that the experimental setups used are ecologically valid. More specifically, if one is interested in the relevance of auditory location processing, it seems necessary to use freefield auditory stimulation comprising the entire extent of spatial cues, instead of relying on artificial headphone presentations with a limited coding of space. This inference seems to be all the more of importance since a substantial part of the research in auditory spatial attention has been based on dichotic listening experiments.

The detection of mismatches differed between the spatialand the identity priming tasks. Whereas Experiment 1 revealed a symmetrical pattern of partial repetition costs

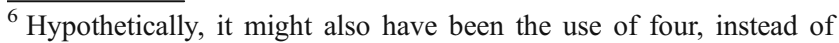
two, locations that was associated with the differences in results between experiments. Although we cannot rule out this alternative explanation on the evidence available, we regard it to be very unlikely. Keep in mind that we argue about location information of the to-beignored prime stimulus, which is incidentally encoded and stored while the participant focuses on the location of the prime target sound. If anything, the use of four locations reduces the spatial discriminability of the incidentally encoded prime distractor sound, as compared with a situation with two locations maximally apart from each other. As a consequence, location encoding and storage should rather decrease than increase in a setup of four possible locations.
} 
for mismatches of both types - changes of the location of a repeated sound and also changes of a sound at a repeated location-Experiment 2 revealed an asymmetrical pattern of partial repetition costs: A mismatch was detected only when the same sound was presented from a different location. In contrast, there was no evidence of a slowdown for mismatches of the other type - that is, when the sound at the repeated location changed between prime and probe.

The different pattern of results in Experiment 1 and Experiment 2 in spite of identical auditory stimulation and response mode clearly indicates the importance of task demands and of the stimulus dimension that is relevant for response generation and, thus, is primarily attended. This conclusion is compatible with a recent finding from a study by Zmigrod and Hommel (2009) showing that features associated with task-relevant stimulus dimensions were more likely to become part of bindings than were taskirrelevant features, suggesting that attention is beneficial to the integration process. However, relevance alone cannot be the whole story, given that we found a symmetry of partial repetition costs in Experiment 1 (both types of identitylocation mismatches impaired responding) but an asymmetry of partial repetition costs in Experiment 2 (only sound repetitions at another location impaired responding). A similar asymmetry between sound identity and sound location has already been reported by Maybery et al. (2009) and also fits with Kubovy and Van Valkenburg's (2001) conceptualization of auditory objects assuming that nonspatial properties are predominant in auditory object formation. However, before we can infer that the differences in the results between Experiment 1 and 2 reflect an asymmetry in auditory memory representations of identity and space, two potential alternative explanations for these differences shall be considered. First, processing speed differences may exist between identity and location information. Second, there may have been compatibility differences between the manual response mode and the chosen tasks.

It is theoretically conceivable that identity and location information were not processed comparably fast in our experiments or-in terms of the related ideas in Melara and Mounts (1993, 1994) - that the stimulus values on the identity dimension and the location dimension were not of comparable discriminability. With respect to processing speed differences, interaction effects between dimensions might appear when the more quickly processed dimension is response irrelevant but interferes, due to its early availability, with processing of the more slowly processed but task-relevant dimension (no interaction would appear the other way round). With respect to stimulus discriminability, Melara and Mounts (1993) demonstrated that an irrelevant dimension interferes more with responding to a relevant dimension if the values of the irrelevant dimension are high in discriminability, relative to the values of the relevant dimension. Turning to our experiments, both identity and location information were relevant in Experiment 1 and 2, although location information was central to responding in Experiment 1, whereas identity information was central to responding in Experiment 2. It seems possible that Experiment 1 required identity processing before location processing ("locate the specified sound"), whereas Experiment 2 required location processing before identity processing ("identify the sound at the specified location"). If, in addition, location information were processed more quickly than identity information, Experiment 1 should suffer more dimensional cross-talk than Experiment 2. This, in turn, might explain the stronger partial repetition costs in Experiment 1 . We think this explanation to be very unlikely for two reasons.

First, although reaction times in Experiment 1 were more than 200 ms shorter, as compared with Experiment 2, this difference cannot be interpreted as an indicator of processing speed differences between location information (central to responding in Experiment 1) and identity information (central to responding in Experiment 2). Obviously, both tasks included identity and location processing. Thus, reaction times cannot be a valid estimate of the processing speed of a single dimension. Instead, the response slowdown in Experiment 2 was due mainly to the more difficult stimulus-response mapping in Experiment 2, as compared with Experiment 1. In other words, the overall response time difference between experiments was probably caused by postperceptual processes, but not by a slowdown in early stimulus processing of identity information, as compared with location information.

Second, although we do not know whether identity and location information were equivalent with respect to processing speed and/or discriminability, we can be sure that perceptual discriminability of the two dimensions was equivalent between experiments, because perceptual aspects of the auditory stimulation were identical in Experiment 1 and 2. As a consequence, differences in the pattern of results must have been caused by differences in task demands only. However, with respect to task demands, it seems very implausible to assume that the findings can be explained by temporal interactions between identity and location processing (as explained above) that might lead to more cross-talk in Experiment 1 than in Experiment 2. If there were such temporal interactions, this would imply that there should be cross-talk only in Experiment 1, but no cross-talk in Experiment 2. However, there was clear crosstalk in the identity task in Experiment 2, but of an asymmetric nature. Participants were sensitive to a repeated sound's changing location. This can imply only that participants processed and stored identity and location 
information together. However, they were insensitive when another sound was presented at a repeated location, probably because location repetition did not function as a retrieval cue for the prime episode when identity was central to the task, as we will argue later on.

The choice of the manual response mode in both experiments is another aspect to which special attention should be paid. We deliberately chose exactly the same stimulus and response layout in both experiments in order to be able to attribute any differences in the results to the differences in the task demands. However, the manual response mode necessarily includes a spatial componentresponse keys have to be spatially aligned-so that response generation in Experiment 2 (identity task) involved a spatial aspect, whereas response generation in Experiment 1 (localization task) did not involve reference to the dimension of identity. Therefore, one could argue that the localization task was compatible with the manual response requirement, whereas the identity task was not (or to a smaller degree). It is difficult to assess what effect this difference in task-response mode compatibility might have had on the differences found between Experiment 1 and 2. However, we have empirical reasons to believe that spatial (in-)compatibility of responses was of very minor importance, if not entirely irrelevant, in Experiment 2, which makes an explanation based on spatial compatibility rather unlikely. In this experiment, each of the four sounds was associated with one of the four response keys. Due to the key layout, each response key was compatible with one of the four loudspeaker positions but incompatible with the three other locations (see Fig. 3). For example, if the bird sound was the target sound, participants had to press the right distal key ("5" on the number block). A trial was response compatible with respect to the stimulus location when the bird sound was presented from the front right loudspeaker. However, when the bird sound was presented from any of the three other locations, stimulus location and response key were incompatible. If this type of compatibility were relevant for responding, we should find different results in Experiment 2 as a function of whether the sound was presented at a location compatible with the correct response or not. We reanalyzed the data separately for compatible and incompatible trials. For both types of trials, we found the same pattern of results as those reported for the analysis of all the trials. We therefore conclude that task-response mode compatibility can be ignored when interpreting the results of Experiment 2. But note that in order to avoid such an additional reanalysis of task-response mode compatibility, a vocal response requirement without a spatial response component would be advisable for future studies comparing localization and identification task performance.

In contrast to an explanation based on processing speed or response mode compatibility, we think that the differen- tial pattern of identity-location mismatch detection found in the two tasks can be understood by assuming two interacting causes: (1) a predisposition in the auditory modality on nonspatial (identity) information in the representation of objects, as has been argued by others before (Maybery et al., 2009; see also Kubovy \& Van Valkenburg, 2001) and was elaborated earlier on in this article, paired with (2) the idea that retrieval routes to previous (i.e., prime) processing episodes stored in memory are shaped by the processing constraints taking place during current (i.e., probe) task processing.

We think that sound identity and location information of ignored sounds is encoded and integrated into processing episodes/object files, even if the whole auditory object is task irrelevant. This is what happened to the prime distractor sounds in both of our tasks, regardless of whether the task focus was on identity or location processing (otherwise, none of the mismatch effects could have been found). We think that the processing focus of a task, however, determines which retrieval cues are effective in cuing previous processing episodes. For example, in our Experiment 2, identity processing was central to the task. As a consequence, effective retrieval cues were restricted to sound identity. Only when the sound identity was repeated could the respective object file from the prime be retrieved. In cases of an identity repetition but a sound location change, the prime object file included different location information, such that identity-location mismatches could cause partial repetition costs. However, when location was repeated but another sound appeared at the same location, this identitylocation mismatch was not detected, because retrieval of the prime object file hinged on sound identity repetition. This explains the asymmetry in partial mismatch effects found in Experiment 2. Note that a related idea has been proposed very recently by Leboe, Leboe, and Milliken (2010). These authors found partial repetition costs in immediate visual-priming tasks to depend on probe task demands. Very similar to our idea, they argued that the processing constraints (i.e., the orienting of attention to a certain stimulus dimension) in the probe task define the retrieval cues to previous (i.e., prime) episodes.

The situation is somewhat different when location is central to the task, as in our Experiment 1. Given the preponderance of identity information in the representation of auditory objects, not only task-relevant location information, but also identity information can function as an effective retrieval cue. Therefore, in the location task, retrieval of object files could occur via two retrieval routes, either identity repetition (as in Experiment 2) or location repetition. When the sound identity was repeated but the location changed, retrieval of the previous prime object file was cued by sound identity. When, however, a different sound 
appeared at the same location, retrieval of the previous prime object file was cued by location. In both cases, the detection of the mismatch was inevitable. This explains the symmetry in the partial mismatch effects found in Experiment 1. In sum, these considerations lead to the conclusion that the differential pattern of results between the two tasks originates from a difference in retrieval cue availability, but not from a difference in object binding. In other words, identity repetitions function by default as a retrieval cue in audition, regardless of task demands. Whether location repetitions additionally cue previous episodes depends on the task relevance of the location dimension.

The supplementary multinomial analyses revealed that prime response retrieval processes (as indicated by an increase of prime response errors) were involved in the identification task in Experiment 2, but not in the localization task in Experiment 1. When a previously ignored sound identity became response relevant in the identity task in Experiment 2, the probability of erroneously repeating the former prime response increased. This finding was in accordance with a number of previous identity priming studies that have revealed an increase in prime response retrieval errors in ignored repetition trials, as compared with control trials (Mayr \& Buchner, 2006, 2010a, 2010b; Mayr et al., 2009a, in press). For the localization task in Experiment 1, however, the probability of prime response retrieval errors was equivalent across all trial types. This result replicated the findings reported by Mayr et al. (2009b), who did not find any evidence of the involvement of prime response retrieval processes in auditory spatial priming. We concluded that actionoriented episodes (event files) may not be stored in the case of location priming, most probably due to the fact that there are no fixed target identity-response bindings established in a spatial task. However, such a binding seems to be necessary to access response information. While this conclusion is in perfect accordance with the findings of Mayr et al., note that this explanation is posthoc and can explain the present results but stays in contrast to retrieval effects of response information as reported by Zmigrod and Hommel (2009; see also Hommel, 2004). These authors found evidence of event files - that is, of stimulus-response integration-both for pitch-response and for location-response bindings. The principle underlying these stimulus-response bindings seemed to be temporal overlap of code activations. Whether there are fundamental differences between the episodic retrieval processes thought to underlie the event file idea in those studies and the episodic retrieval processes thought to underlie the prime response retrieval effects found in our auditory negative priming task cannot be answered to date.

In closing, the experiments reported here demonstrate that auditory identity and location negative priming share underlying processes - that is, the detection of identitylocation mismatches. At the same time, the experiments show that identity and location negative priming differ in the degree to which mismatch detection, as well as retrieval of response information, is involved.

Author Notes Susanne Mayr, Axel Buchner, Malte Möller, and Robert Hauke, Department of Experimental Psychology, Heinrich-HeineUniversität Düsseldorf, Germany. The research reported in this article was supported by a grant from the Deutsche Forschungsgemeinschaft (Ma 2610/2-2) and is part of the habilitation thesis of the first author. We thank Alina Ignaz for her assistance with data collection.

\section{References}

Banks, W. P., Roberts, D., \& Ciranni, M. (1995). Negative priming in auditory attention. Journal of Experimental Psychology. Human Perception and Performance, 21, 1354-1361.

Buchner, A., \& Mayr, S. (2004). Auditory negative priming in younger and older adults. The Quarterly Journal of Experimental Psychology, 57A, 769-787.

Buchner, A., \& Steffens, M. C. (2001). Auditory negative priming in speeded reactions and temporal order judgements. The Quarterly Journal of Experimental Psychology, 54A, 1125-1142.

Buchner, A., Zabal, A., \& Mayr, S. (2003). Auditory, visual, and cross-modal negative priming. Psychonomic Bulletin \& Review, 10, 917-923.

Buckolz, E., Avramidis, C., \& Fitzgeorge, L. (2008). Prime-trial processing demands and their impact on distractor processing in a spatial negative priming task. Psychological Research/Psychologische Forschung, 72, 235-248.

Buckolz, E., Goldfarb, A., \& Khan, M. (2004). The use of a distractorassigned response slows later responding in a location negative priming task. Perception \& Psychophysics, 66, 837-845.

Chao, H.-F. (2009). Revisiting the role of probe distractors in negative priming: Location negative priming is observed when probe distractors are consistently absent. Attention, Perception, \& Psychophysics, 71, 1072-1082.

Dyson, B. J., \& Ishfaq, F. (2008). Auditory memory can be object based. Psychonomic Bulletin \& Review, 15, 409-412.

Eimer, M., \& Schlaghecken, F. (1998). Effects of masked stimuli on motor activation: Behavioral and electrophysiological evidence. Journal of Experimental Psychology. Human Perception and Performance, 24, 1737-1747.

Faul, F., Erdfelder, E., Lang, A.-G., \& Buchner, A. (2007). G*Power 3: A flexible statistical power analysis program for the social, behavioral, and biomedical sciences. Behavior Research Methods, 39, 175-191.

Fitzgeorge, L., \& Buckolz, E. (2008). Spatial negative priming modulation: The influence of probe-trial target cueing, distractor presence, and an intervening response. European Journal of Cognitive Psychology, 20, 994-1026.

Fletcher, B. C., \& Rabbitt, P. M. (1978). The changing pattern of perceptual analytic strategies and response selection with practice in a two-choice reaction time task. The Quarterly Journal of Experimental Psychology, 30, 417-427.

Fox, E. (1995). Negative priming from ignored distractors in visual selection: A review. Psychonomic Bulletin \& Review, 2, 145-173.

Gibbons, H. (2006). An event-related potential investigation of varieties of negative priming. Journal of Psychophysiology, 20, $170-185$. 
Guy, S., \& Buckolz, E. (2007). The locus and modulation of the location negative priming effect. Psychological Research, 71, $178-191$

Guy, S., Buckolz, E., \& Khan, M. (2006). The locus of location repetition latency effects. Canadian Journal of Experimental Psychology, 60, 307-318.

Guy, S., Buckolz, E., \& Pratt, J. (2004). The influence of distractoronly prime trials on the location negative priming mechanism. Experimental Psychology, 51, 4-14.

Hall, M. D., Pastore, R. E., Acker, B. E., \& Huang, W. (2000). Evidence for auditory feature integration with spatially distributed items. Perception \& Psychophysics, 62, 1243-1257.

Holm, S. (1979). A simple sequentially rejective multiple test procedure. Scandinavian Journal of Statistics, 6, 65-70.

Hommel, B. (2004). Event files: feature binding in and across perception and action. Trends in Cognitive Sciences, 8, 494500

$\mathrm{Hu}$, X., \& Batchelder, W. H. (1994). The statistical analysis of engineering processing tree models with the EM algorithm. Psychometrika, 59(1), 21-47.

Huang, L., Holcombe, A. O., \& Pashler, H. (2004). Repetition priming in visual search: Episodic retrieval, not feature priming. Memory \& Cognition, 32, 12-20.

Kahneman, D., Treisman, A., \& Gibbs, B. J. (1992). The reviewing of object files: Object-specific integration of information. Cognitive Psychology, 24, 175-219.

Krueger, L. E., \& Shapiro, R. G. (1981). Intertrial effects of same different judgements. The Quarterly Journal of Experimental Psychology, 33A, 241-265.

Kubovy, M., \& Van Valkenburg, D. (2001). Auditory and visual objects. Cognition, 80, 97-126.

Leboe, J. P., Leboe, L. C., \& Milliken, B. (2010). Constraints on the observation of partial match costs: Implications for transferappropriate processing approaches to immediate priming. Journal of Experimental Psychology. Human Perception and Performance, 36, 634-648.

Leboe, J. P., Mondor, T. A., \& Leboe, L. C. (2006). Feature mismatch effects in auditory negative priming: Interference as dependent on salient aspects of prior episodes. Perception \& Psychophysics, $68,897-910$

Maybery, M. T., Clissa, P. J., Parmentier, F. B. R., Leung, D., Harsa, G., Fox, A. M., et al. (2009). Binding of verbal and spatial features in auditory working memory. Journal of Memory and Language, 61, 112-133.

Mayr, S., \& Buchner, A. (2006). Evidence for episodic retrieval of inadequate prime responses in auditory negative priming. Journal of Experimental Psychology. Human Perception and Performance, 32, 932-943.

Mayr, S., \& Buchner, A. (2007). Negative priming as a memory phenomenon: A review of 20 years of negative priming research. Zeitschrift für Psychologie/Journal of Psychology, $215,35-51$

Mayr, S., \& Buchner, A. (2010a). Auditory negative priming endures response modality change; prime-response retrieval does not. The Quarterly Journal of Experimental Psychology, 63, 653-665.

Mayr, S., \& Buchner, A. (2010b). Episodic retrieval processes take place automatically in auditory negative priming. European Journal of Cognitive Psychology, 22, 1192-1221.

Mayr, S., Buchner, A., \& Dentale, S. (2009a). Prime retrieval of motor responses in negative priming. Journal of Experimental Psychology. Human Perception and Performance, 35, 408-423.

Mayr, S., Hauke, R., \& Buchner, A. (2009b). Auditory location negative priming: A case of feature mismatch. Psychonomic Bulletin \& Review, 16, 845-849.
Mayr, S., Hauke, R., Buchner, A., \& Niedeggen, M. (2009c). No evidence for a cue mismatch in negative priming. The Quarterly Journal of Experimental Psychology, 62, 645-652.

Mayr, S., Möller, M., \& Buchner, A. (in press). Evidence of vocal and manual event files in auditory negative priming. Experimental Psychology.

Mayr, S., Niedeggen, M., Buchner, A., \& Orgs, G. (2006). The level of reaction time determines the ERP correlates of auditory negative priming. Journal of Psychophysiology, 20, 186-194.

Melara, R. D., \& Mounts, J. R. (1993). Selective attention to Stroop dimensions: Effects of baseline discriminability, response mode, and practice. Memory \& Cognition, 21, 627-645.

Melara, R. D., \& Mounts, J. R. W. (1994). Contextual influences on interactive processing: Effects of discriminability, quantity, and uncertainty. Perception \& Psychophysics, 56, 73-90.

Milliken, B., Tipper, S. P., Houghton, G., \& Lupiáñez, J. (2000). Attending, ignoring, and repetition: On the relation between negative priming and inhibition of return. Perception \& Psychophysics, 62, 1280-1296.

Milliken, B., Tipper, S. P., \& Weaver, B. (1994). Negative priming in a spatial localization task: Feature mismatching and distractor inhibition. Journal of Experimental Psychology. Human Perception and Performance, 20, 624-646.

Mondor, T. A., \& Breau, L. M. (1999). Facilitative and inhibitory effects of location and frequency cues: Evidence of a modulation in perceptual sensitivity. Perception \& Psychophysics, 61, 438-444.

Mondor, T. A., Breau, L. M., \& Milliken, B. (1998a). Inhibitory processes in auditory selective attention: Evidence of locationbased and frequency-based inhibition of return. Perception \& Psychophysics, 60, 296-302.

Mondor, T. A., Leboe, J. P., \& Leboe, L. C. (2005). The role of selection in generating auditory negative priming. Psychonomic Bulletin \& Review, 12, 289-294.

Mondor, T. A., Zatorre, R. J., \& Terrio, N. A. (1998b). Constraints on the selection of auditory information. Journal of Experimental Psychology. Human Perception and Performance, 24, 66-79.

Neill, W. T., \& Valdes, L. A. (1992). Persistence of negative priming: Steady state or decay? Journal of Experimental Psychology. Learning, Memory, and Cognition, 18, 565-576.

Neill, W. T., Terry, K. M., \& Valdes, L. A. (1994). Negative priming without probe selection. Psychonomic Bulletin \& Review, 1, 119-121.

Neill, W. T., Valdes, L. A., Terry, K. M., \& Gorfein, D. S. (1992). Persistence of negative priming: II. Evidence for episodic trace retrieval. Journal of Experimental Psychology. Learning, Memory, and Cognition, 18, 993-1000.

Park, J., \& Kanwisher, N. (1994). Negative priming for spatial locations: Identity mismatching, not distractor inhibition. Journal of Experimental Psychology. Human Perception and Performance, 20, 613-623.

Parmentier, F. B. R., Maybery, M. T., \& Elsley, J. (2010). The involuntary capture of attention by novel feature pairings: A study of voice-location integration in auditory sensory memory. Attention, Perception, \& Psychophysics, 72, 279-284.

Pashler, H. E., \& Baylis, G. C. (1991). Procedural learning: II. Intertrial repetition effects in speeded-choice tasks. Journal of Experimental Psychology. Learning, Memory, and Cognition, 17, $33-48$.

Prime, D. J., \& Ward, L. M. (2002). Auditory frequency-based inhibition differs from spatial IOR. Perception \& Psychophysics, 64, 771-784.

Reuter-Lorenz, P. A., Jha, A. P., \& Rosenquist, J. N. (1996). What is inhibited in inhibition of return? Journal of Experimental Psychology. Human Perception and Performance, 22, 367-378. 
Roberts, K. L., Summerfield, A. Q., \& Hall, D. A. (2009). Covert auditory spatial orienting: An evaluation of the spatial relevance hypothesis. Journal of Experimental Psychology. Human Perception and Performance, 35, 1178-1191.

Rothermund, K., Wentura, D., \& De Houwer, J. (2005). Retrieval of incidental stimulus-response associations as a source of negative priming. Journal of Experimental Psychology. Learning, Memory, and Cognition, 31, 482-495.

Rothkegel, R. (1999). AppleTree: A multinomial processing tree modeling program for Macintosh computers. Behavior Research Methods, Instruments, \& Computers, 31(4), 696-700.

Tipper, S. P. (1985). The negative priming effect: Inhibitory priming by ignored objects. The Quarterly Journal of Experimental Psychology, 37A, 571-590.

Tipper, S. P. (2001). Does negative priming reflect inhibitory mechanisms? A review and integration of conflicting views. The Quarterly Journal of Experimental Psychology, 54A, 321-343.

Tipper, S. P., \& Cranston, M. (1985). Selective attention and priming: Inhibitory and facilitatory effects of ignored primes. The Quarterly Journal of Experimental Psychology, 37A, 591-611.

Tipper, S. P., Brehaut, J. C., \& Driver, J. (1990). Selection of moving and static objects for the control of spatially directed action. Journal of Experimental Psychology. Human Perception and Performance, 16, 492-504.

Tipper, S. P., Weaver, B., \& Milliken, B. (1995). Spatial negative priming without mismatching: Comment on Park and Kanwisher (1994). Journal of Experimental Psychology. Human Perception and Performance, 21, 1220-1229.

Zabal, A., \& Buchner, A. (2006). Normal auditory negative priming in schizophrenic patients. The Quarterly Journal of Experimental Psychology, 59, 1224-1236.

Zmigrod, S., \& Hommel, B. (2009). Auditory event files: Integrating auditory perception and action planning. Attention, Perception, \& Psychophysics, 71, 352-362.

Zmigrod, S., \& Hommel, B. (2010). Temporal dynamics of unimodal and multimodal feature binding. Attention, Perception, \& Psychophysics, 72, 142-152. 